\title{
Apico-basal forces exerted by apoptotic cells drive epithelium folding
}

Bruno Monier ${ }^{1,2 *}$, Melanie Gettings ${ }^{1,2 *}$, Guillaume Gay $^{3}$, Thomas Mangeat ${ }^{1,2}$, Sonia Schott ${ }^{1,2}$, Ana Guarner ${ }^{4}$ \& Magali Suzanne ${ }^{1,2}$

\begin{abstract}
Epithelium folding is a basic morphogenetic event that is essential in transforming simple two-dimensional epithelial sheets into threedimensional structures in both vertebrates and invertebrates ${ }^{1}$. Folding has been shown to rely on apical constriction ${ }^{2-7}$. The resulting cell-shape changes depend either on adherens junction basal shift ${ }^{2}$ or on a redistribution of myosin $\mathrm{II}^{3-5,7}$, which could be driven by mechanical signals ${ }^{8}$. Yet the initial cellular mechanisms that trigger and coordinate cell remodelling remain largely unknown. Here we unravel the active role of apoptotic cells in initiating morphogenesis, thus revealing a novel mechanism of epithelium folding. We show that, in a live developing tissue, apoptotic cells exert a transient pulling force upon the apical surface of the epithelium through a highly dynamic apico-basal myosin II cable. The apoptotic cells then induce a non-autonomous increase in tissue tension together with cortical myosin II apical stabilization in the surrounding tissue, eventually resulting in epithelium folding. Together our results, supported by a theoretical biophysical three-dimensional model, identify an apoptotic myosin-II-dependent signal as the initial signal leading to cell reorganization and tissue folding. This work further reveals that, far from being passively eliminated as generally assumed (for example, during digit individualization ${ }^{9}$ ), apoptotic cells actively influence their surroundings and trigger tissue remodelling through regulation of tissue tension.
\end{abstract}

In different morphogenetic contexts, apoptosis has been shown to have an essential role in tissue folding; however, the cellular mechanisms involved remain mostly unknown ${ }^{10-12}$. To characterize apoptosisdependent folding, we focused on Drosophila leg epithelium morphogenesis, a process that has been shown to rely on local apoptosis ${ }^{11}$ (Extended Data Fig. 1a, Supplementary Video 1). Interestingly, we discovered that leg disc folding follows a stereotypical sequence, with fold progression following the spreading of cell death, beginning in the most ventral part, then progressing laterally to end in the most dorsal region of the developing leg (Fig. 1a, b, Extended Data Fig. 1b). To unravel the link between apoptotic cells and fold formation, we first focused on apoptotic cell behaviour using live imaging. In the leg epithelium, apoptosis follows the classical morphological steps including cell shrinkage, membrane blebbing and fragmentation into apoptotic bodies (Extended Data Fig. 1c). Initially, apoptotic cells remain columnar and attached to their neighbours, as described previously ${ }^{13}$ (Extended Data Fig. 2a). Indeed, we noticed that adherens junction components (E-cadherin, $\alpha$-catenin and $\beta$-catenin) accumulate below the apical surface of dying cells, forming an adhesion peak which coincides with local deformation of the apical surface of the surrounding epithelial cells (Fig. 1c, Supplementary Video 2). These observations prompted us to hypothesize either the presence of an apico-basal pulling force generated by the dying cells or, alternatively, a pushing force generated by the dying cell's neighbours. Therefore, we analysed myosin II dynamics. Interestingly, we detected an apico-basal acto-myosin cable-like structure (hereafter named 'cable') inside each dying cell (Extended Data Fig. 2b) that is formed just before the local deformation of the epithelium surface (Fig. 1d, Supplementary Video 3). This myosin II cable is attached to the junctional structure described above (Fig. 1e). Remarkably, apical surface release coincides with myosin II cable and adhesion peak detachment from the apical surface as the dying cell fragments (Fig. 1c, d, Extended Data Fig. 2c, d). Furthermore, when apoptosis is inhibited, neither the myosin II cable nor the apical deformation are observed (Extended Data Fig. 2e), suggesting that the myosin II cable constitutes the cellular apoptotic machinery responsible for the transient deformation of the epithelium.

We then asked whether the apico-basal myosin II cable is a general characteristic of apoptotic epithelial cells. By analysing myosin II distribution in different Drosophila epithelial tissues, we revealed that an apico-basal myosin II cable also forms in apoptotic cells in other epithelia (Extended Data Fig. 2h).We further asked if this general property of apoptotic cells to generate an apico-basal myosin II cable is responsible for the local apical deformation observed around apoptotic cells. To test this, we induced ectopic apoptosis in the Drosophila wing which can be regarded as a naive tissue (as apoptosis normally occurs sporadically) and blocked myosin II function specifically in dying cells. While an apico-basal myosin II cable is formed in ectopic dying cells (Extended Data Fig. 2f), the local apical deformation around apoptotic cells is no longer visible when myosin II is inhibited (compare Fig. If to Fig. 1g, Extended Data Fig. 2g), indicating that the deformation strictly results from the myosin II dependent apoptotic force and is not generated by neighbouring cells. Together, these experiments demonstrate a fundamental intrinsic in vivo property of yet non-fragmented apoptotic cells, namely their ability to produce a myosin II dependent apico-basal pulling force capable of transiently deforming adjacent cells (Fig. 1h).

To determine how apoptotic cells control the reorganization of the remaining tissue, we characterized myosin II distribution and cell shape changes in the vicinity of apoptotic cells. Myosin II in this tissue is localized at the level of adherens junctions (Extended Data Fig. 3a, b). Interestingly, we observed that myosin II levels are increased along the apical membrane of apoptotic cell neighbours (Fig. 2a). Eventually, myosin II and F-actin apical stabilization is found throughout the whole fold domain where apoptosis takes place compared to the segment domain (Fig. 2b, Extended Data Fig. 3c, d) and is lost in absence of apoptosis (Fig. 2c and Extended Data Fig. 3e-g). Moreover, we observed that cells neighbouring apoptotic cells become progressively elongated and reduce their apical surface (Fig. 2d, Supplementary Video 4). Elongation then propagates from cell to cell, gradually spreading to the whole fold domain, thus generating a ring of stretched cells in which apoptosis occurs specifically (Extended Data Fig. 4a, b, Supplementary Video 5). These observations suggest that cell death is responsible for cell shape modification in the whole fold domain. We therefore compared cell shape dynamics of developing legs with or without apoptosis. In the control fold domain, cells elongate, decrease their apical surface and adopt a preferential orientation along the future fold (Fig. 2e). However, all these characteristics are lost when cell death is inhibited, demonstrating the essential role of apoptosis in determining cell morphology during

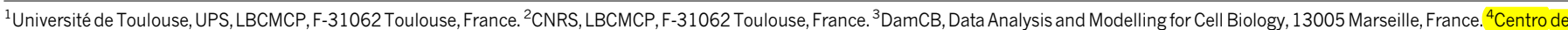
Biología Molecular Severo Ochoa (C.S.I.C.-U.A.M.), Universidad Autónoma de Madrid, Nicolás Cabrera 1, Cantoblanco, 28049 Madrid, Spain.

*These authors contributed equally to this work. 
a

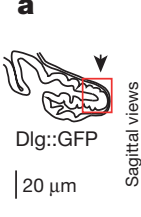

b
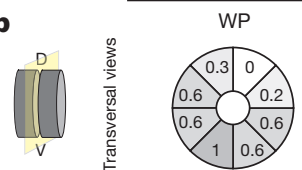

Pre-fold stage

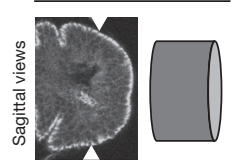

$W P+1 \mathrm{~h}$

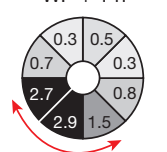

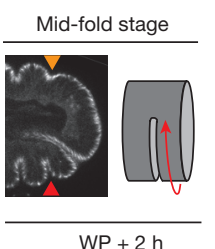

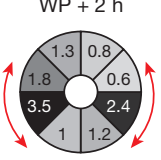

Late-fold stage

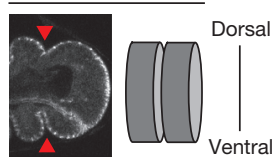

$W P+3 h$ e

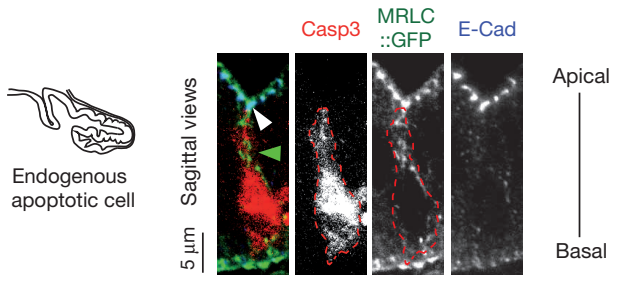

f

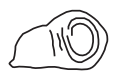

Ectopic apoptotic cell g

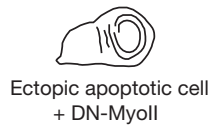

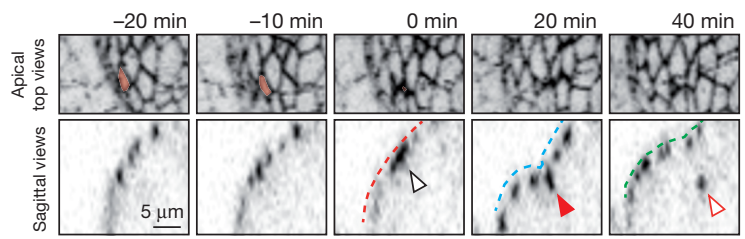

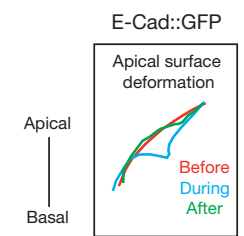

d
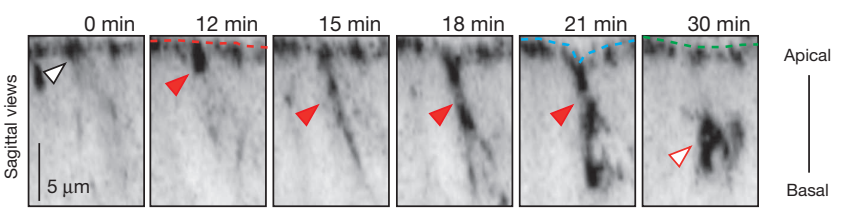

Figure $1 \mid$ Apoptotic cells exert a transient apico-basal force upon adjacent cells. a, Pupal leg disc from pre-fold stage (white pupae, WP) to late-fold stage $(\mathrm{WP}+3 \mathrm{~h})$. Arrowhead colours indicate fold progression $(n=10,7,10$ and 10, respectively). b, Average number of dying cells in the fold domain $(n=10,6$, 10 and 7, respectively). A, anterior; P, posterior. c, d, E-cadherin-green fluorescent protein (E-Cad::GFP) $(\mathbf{c}, n=24)$ and MRLC::GFP (d, $n=21)$ dynamics in apoptotic cells (in red in c) from pre-fold stage leg discs. Time $0=$ collapse of apoptotic cell apical surface; the dotted line and arrowhead

folding (Fig. 2e and Extended Data Fig. 4c-e). Using laser ablation, we tested whether cell elongation and myosin II stabilization at the level of adherens junctions reveal an increase in tissue tension in the fold domain, as previously described in other tissues ${ }^{14}$. We found that the release of tension between vertices was indeed much higher in the fold domain where apoptosis takes place than in the segment domain (Extended Data Fig. 3 h). In addition, when cell death was inhibited, tension

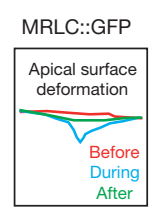

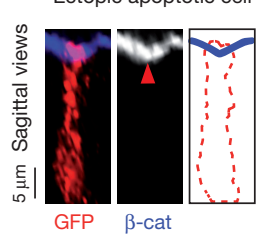

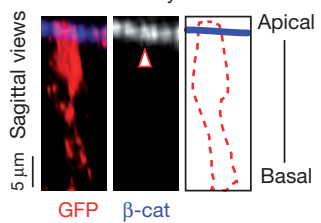

h

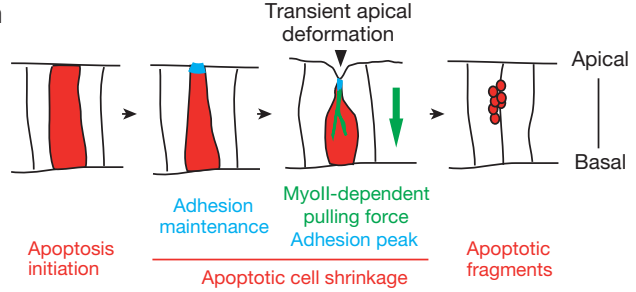

colour codes indicate equivalent stages of the apoptotic process. e, Colocalization of the apoptotic myosin II cable (green arrowhead) with adherens junctions ( $n=19$, white arrowhead) stained with anti-E-Cad. f, g, Ectopic apoptotic cells with (f) or without (g) myosin II activity generated in the wing disc. DN, dominant negative form. Red and open arrowheads point at presence or absence of apical deformation, respectively (see quantification and genotypes in Extended Data Fig. 2g). h, Schematics of apoptotic cell dynamics.

in the fold domain was significantly lower than in the control situation (Extended Data Fig. 3i). Altogether these results demonstrate that, during leg folding, apoptosis induces a non-autonomous effect throughout the fold domain leading to acto-myosin apical stabilization, a global enhancement of tissue tension and cell shape changes (Fig. 2f).

To test the role of apoptotic forces in folding, we constructed a physical model based on the two-dimensional vertex model $^{15}$. In this model,

a

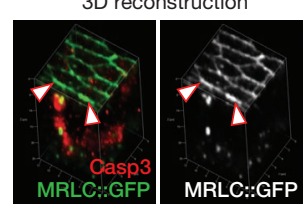

b

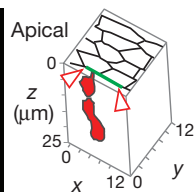

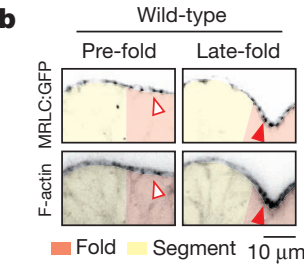

c

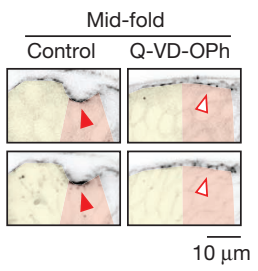

d
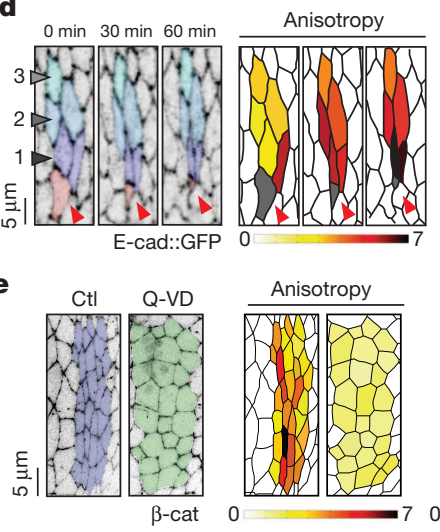

Anisotropy

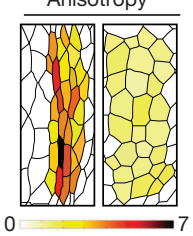

Area $\left(\mu \mathrm{m}^{2}\right)$
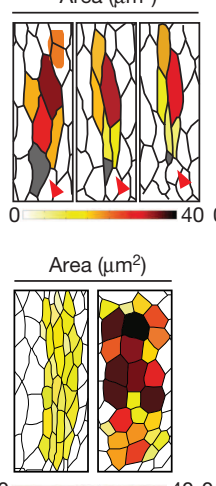

Orientation $\left(^{\circ}\right)$

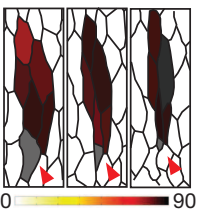

Orientation $\left({ }^{\circ}\right)$

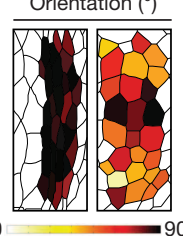

$\mathbf{f}$ transmission
of apoptotic force
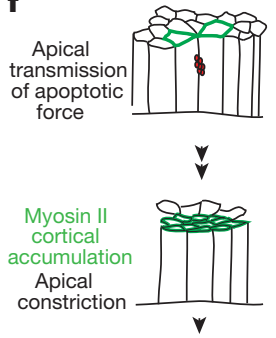

Tissue folding
Apical
transmission
Figure $2 \mid$ The apoptotic force propagates to the neighbouring tissue. a, Leg disc threedimensional reconstruction and schematics showing apical myosin II stabilization (arrowheads) in cells adjacent to a dead cell $(n=11)$. b, Leg discs from pre-fold stage $(n=9)$ and late-fold stage $(n=7)$. c, Leg discs incubated from pre-fold to mid-fold stages with DMSO (control, $n=13$ ) or Q-VD-OPh (cell death inhibition, $n=13$ ). Red and open arrowheads point to the presence or absence, respectively, of F-actin or myosin II stabilization in $\mathbf{b}$ and $\mathbf{c}$. d, Leg disc time-lapse images and quantification of cell shape change parameters at the vicinity of an apoptotic cell (red arrowheads, $n=18$ ). e, Quantification of cell shape change parameters of leg discs incubated with DMSO $(n=7)$ or Q-VD$\mathrm{OPh}(n=8)$ from pre-fold to mid-fold stages. f, Schematics of apoptotic force propagation. 
a
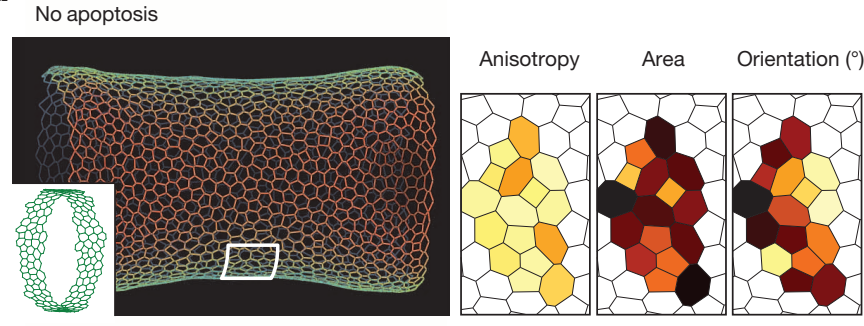

b

Apoptosis without apoptotic forces
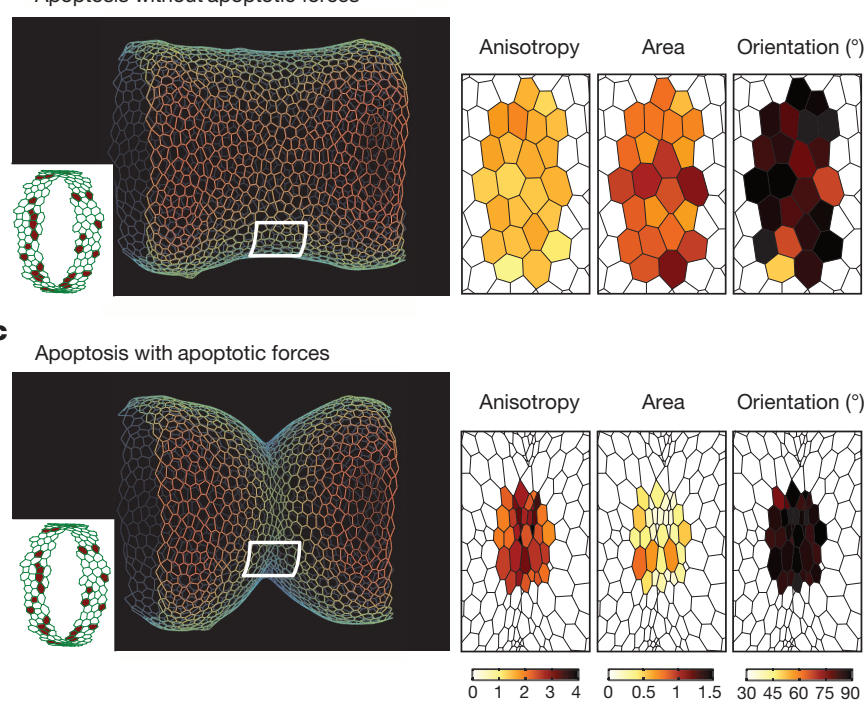

Figure 3 Biophysical model of epithelium folding. a-c, In silico models showing apoptotic pattern (insets), whole tissue (left) and cell shape (right, including normalized area) in the absence of apoptosis (a) and after removing apoptotic cells from the fold domain without (b) and with (c) an apico-basal force generated by each dying cell and transmitted apically to neighbours (noted 'apoptotic forces').

three interactions are considered: cell elasticity dependent on the cell apical area, a contractility term dependent on the cell perimeter, and line tension dependent on apical junction length. To take into account all three dimensions of the leg epithelium, we have added an apicobasal tension to those interactions (Extended Data Fig. 5a), and have considered cell elasticity as a function of cell volume rather than cell area while retaining the main characteristics of the original model (Extended Data Fig. 5b-e). Based on our observation of the developing leg disc, the leg tissue in our model is represented by a 50 -cell circumferential cylinder, with the fold domain representing three rings of cells in which 30 cells are programmed to die following the pattern of apoptosis in the leg (compare the cell death pattern in Fig. $1 \mathrm{~b}$ and Extended Data Fig. 6a). In the absence of the apoptotic-dependent forces, the model indicates that the simple disappearance of 30 cells from a continuous ring-like domain is not sufficient to induce cell shape reorganization and to create an invagination at the tissue level (compare Fig. 3a and b). However, if a transient apico-basal force is applied in each dying cell, an invagination response is observed all around the cylindrical tissue, albeit irregular (Extended Data Fig. 8a). Now, if an increase of apical contractility is applied in two rows of apoptotic neighbours (representing the non-autonomous increase in tissue tension), a cell shape reorganization is observed, although moderate (Extended Data Fig. 7a). Finally, if the transient pulling forces generated at a cellular scale are translated as increased contractility at the tissue scale we observe cell shape reorganization similar to that observed in the leg fold, along with regular and deeper folding (Fig. 3c). This shows that the added effect of both apoptotic forces is necessary and sufficient to induce folding in silico. Importantly, rising apoptotic cell number (Extended Data Fig. 6), apicobasal force strength (Extended Data Fig. 7) or the increase in apical contractility in apoptotic cells neighbours (Extended Data Fig. 8) leads to a gradation in cell and tissue shape changes and demonstrates the robustness of the model.

Furthermore, our model predicts that an apico-basal pulling force generated by sporadic apoptosis is not sufficient to modify tissue shape (Extended Data Fig. 6c, d). Moreover, the synergy of forces arising from several apoptotic cells concentrated in a restricted region appears necessary to generate a force strong enough to produce a fold (compare Extended Data Fig. 6 f with Extended Data Fig. 6g). To test this in vivo we induced apoptosis ectopically in the wing pouch (that is, a flat tissue) and observed that a high concentration of apoptotic cells in a restricted region is indeed sufficient to drastically modify the shape of the epithelium through the creation of an ectopic fold (Fig. 4a, b, left and middle panels, and Extended Data Fig. 9a-b'). Consistently, no folding was observed in regions where only sporadic apoptotic cells were generated (Extended Data Fig. 9b" ' ). Importantly, this tissue bending coincides with an apical stabilization of myosin II (Fig. 4c, middle panel, Extended Data Fig. 9d-e' ) and F-actin (Extended Data Fig. 9c'). It also strictly relies on apoptotic myosin II since the expression of a dominant a
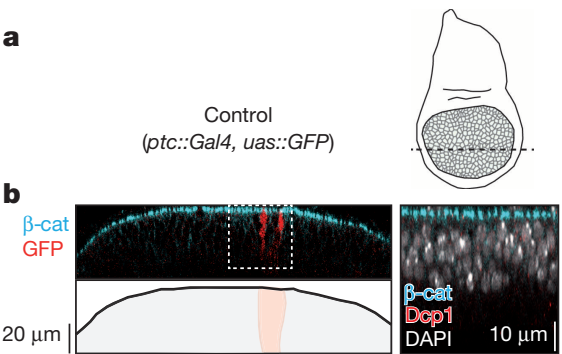

c
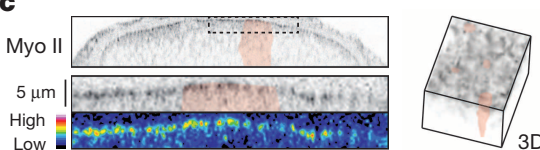

Ectopic cell death (ptc::Gal4, uas::GFP $\times$ uas::GFP, uas:::rpr)
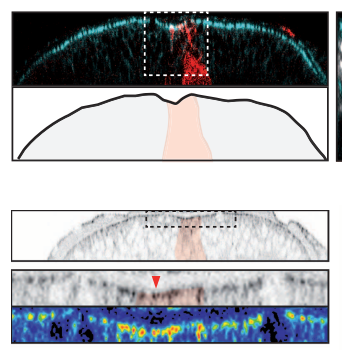
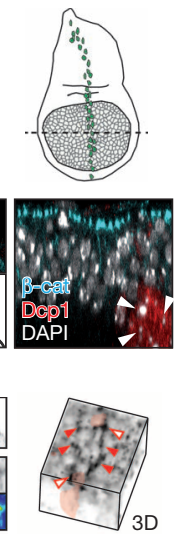

Ectopic cell death + DN-Myoll (ptc::Gal4, uas::GFP $\times$ uas::DN-zip-GFP, uas:::rpr)
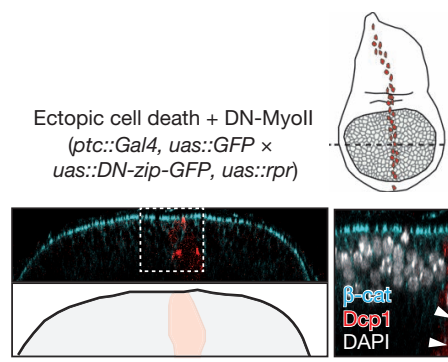

Apoptotic cells with normal Myoll activity

Apoptotic cells with inhibition of Myoll activity
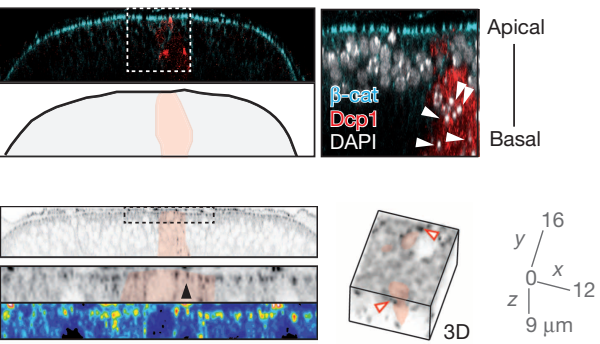

Figure $4 \mid$ Ectopic fold formation. a, Schematics of wing discs depicting the genetic contexts analysed in $\mathbf{b}$ and $\mathbf{c} . \mathbf{b}, \mathbf{c}$, Wing discs in the absence (left panels) or presence (middle and right panels) of ectopic apoptosis in the ptc domain (pink), with (middle panels) or without (right panels) myosin II activity in dying cells, showing wing disc morphogenesis (large panels in $\mathbf{b}$ ) and dying cell extrusion (close ups in $\mathbf{b}$, white arrowheads) and myosin II distribution (stained by an anti-Sqh/MRLC, $\mathbf{c}$; in sagittal sections, red and black arrowheads point to the presence or absence, respectively, of myosin II apical stabilization). Close-up three-dimensional reconstructions are presented in $\mathbf{c}$ to visualize the different pools of apical myosin II and apoptotic cells; red and open arrowheads denote 'fold domain apical myosin II' and 'contractile ring of myosin II', respectively (see also Extended Data Fig. 9e-e' ${ }^{\prime \prime}$ ). b, $n=8,13$ and 10; c, $n=6,7$ and 8 in left, middle and right panels, respectively. 
negative form of myosin II specifically in dying cells at the onset of apoptosis induction suppresses folding (Fig. 4a, b, right panels, and Extended Data Fig. 9a' $\left.\mathrm{a}^{\prime \prime}, \mathrm{b}^{\prime \prime}\right)$, whereas apoptotic cell extrusion from the epithelium remains normal (Fig. $4 \mathrm{~b}$, middle and right panels). Interestingly, the absence of fold in this context is concomitant with an absence of myosin II apical stabilization in the whole domain of ectopic apoptosis (although the myosin II contractile ring involved in dying cell extrusion ${ }^{16}$ is still present, see right panel in Fig. $4 \mathrm{c}$ and Extended Data Fig. $\left.9 \mathrm{e}^{\prime \prime}\right)$, showing that this accumulation strictly depends on apoptotic myosin II (compare Fig. 4c middle panel with right panel, quantifications are in Extended Data Fig. 9d). Altogether, these data strongly suggest that the apoptotic force resulting from the synergy of numerous and patterned apoptotic events constitutes the primary signal leading to epithelium folding.

In this study, we elucidated a novel cellular mechanism of epithelial folding that relies on apoptosis. In Drosophila epithelia, cells are extremely columnar and apoptotic cells, in addition to the force generated by their extrusion from the epithelium as previously described in squamous epithelium ${ }^{17}$, create a myosin-II-dependent apico-basal intracellular pulling force. Based on previous publications showing that transient mechanical forces are sufficient to induce myosin II recruitment at the apical surface of an epithelium ${ }^{8,14}$, we propose that apoptotic cells send a biomechanical signal to their neighbours, although we cannot exclude the myosin-II-dependent release of a molecular signal. The apoptotic signal triggers non-autonomous myosin II recruitment at the level of adherens junctions of neighbouring cells. The synergy of several apoptotic cells leads to a redistribution of myosin II, increased tension, apical constriction in the entire fold domain and subsequent tissue folding (Extended Data Fig. 10). Taken together, these results reveal that through the regulation of tissue tension, apoptotic cells can actively control tissue remodelling.

Interestingly, apoptosis-dependent folding has also been described in vertebrates during neural tube bending. This bending is an important step in neural tube closure and its failure can lead to spina bifida phenotypes ${ }^{12,18}$. Therefore, it would be interesting to test if the cellular mechanisms described here are conserved in vertebrates, thus generalizing the mechanism of apoptosis-dependent epithelium folding.

Online Content Methods, along with any additional Extended Data display items and Source Data, are available in the online version of the paper; references unique to these sections appear only in the online paper.

Received 10 December 2013; accepted 9 December 2014.

Published online 21 January 2015.

1. Davidson, L. A. Epithelial machines that shape the embryo. Trends Cell Biol. 22, 82-87 (2012)

2. Wang, Y. C., Khan, Z., Kaschube, M. \& Wieschaus, E. F. Differential positioning of adherens junctions is associated with initiation of epithelial folding. Nature $\mathbf{4 8 4}$, 390-393 (2012).

3. Martin, A. C., Kaschube, M. \& Wieschaus, E. F. Pulsed contractions of an actin-myosin network drive apical constriction. Nature 457, 495-499 (2009).
4. Roh-Johnson, M. et al. Triggering a cell shape change by exploiting preexisting actomyosin contractions. Science 335, 1232-1235 (2012).

5. Sherrard, K., Robin, F., Lemaire, P. \& Munro, E. Sequential activation of apical and basolateral contractility drives ascidian endoderm invagination. Curr. Biol. 20, 1499-1510 (2010).

6. He, B., Doubrovinski, K., Polyakov, O. \& Wieschaus, E. Apical constriction drives tissue-scale hydrodynamic flow to mediate cell elongation. Nature $\mathbf{5 0 8 , 3 9 2 - 3 9 6}$ (2014).

7. Brodu, V. \& Casanova, J. The RhoGAP crossveinless-c links trachealess and EGFR signaling to cell shape remodeling in Drosophila tracheal invagination. Genes Dev. 20, 1817-1828 (2006)

8. Pouille, P.A., Ahmadi, P., Brunet, A. C. \& Farge, E. Mechanical signals trigger myosin II redistribution and mesoderm invagination in Drosophila embryos. Sci. Signal. 2, ra16 (2009).

9. Montero, J. A. \& Hurlé, J. M. Sculpturing digit shape by cell death. Apoptosis 15, 365-375 (2010).

10. Lohmann, I., McGinnis, N., Bodmer, M. \& McGinnis, W. The Drosophila Hox gene deformed sculpts head morphology via direct regulation of the apoptosis activator reaper. Cell 110, 457-466 (2002).

11. Manjón, C., Sánchez-Herrero, E. \& Suzanne, M. Sharp boundaries of Dpp signalling trigger local cell death required for Drosophila leg morphogenesis. Nature Cell Biol. 9, 57-63 (2007)

12. Yamaguchi, Y. et al. Live imaging of apoptosis in a novel transgenic mouse highlights its role in neural tube closure. J. Cell Biol. 195, 1047-1060 (2011).

13. Lubkov, V. \& Bar-Sagi, D. E-cadherin-mediated cell coupling is required for apoptotic cell extrusion. Curr. Biol. 24, 868-874 (2014).

14. Fernandez-Gonzalez, R., Simoes, S. M., Röper, J. C., Eaton, S. \& Zallen, J. A. Myosin II dynamics are regulated by tension in intercalating cells. Dev. Cell 17, 736-743 (2009).

15. Farhadifar, R., Röper, J. C., Aigouy, B., Eaton, S. \& Jülicher, F. The influence of cell mechanics, cell-cell interactions, and proliferation on epithelial packing. Curr. Biol. 17, 2095-2104 (2007)

16. Rosenblatt, J., Raff, M. C. \& Cramer, L. P. An epithelial cell destined for apoptosis signals its neighbors to extrude it by an actin-and myosin-dependent mechanism. Curr. Biol. 11, 1847-1857 (2001).

17. Toyama, Y., Peralta, X. G., Wells, A. R., Kiehart, D. P. \& Edwards, G. S. Apoptotic force and tissue dynamics during Drosophila embryogenesis. Science 321, 1683-1686 (2008).

18. Yamaguchi, Y. \& Miura, M. How to form and close the brain: insight into the mechanism of cranial neural tube closure in mammals. Cell. Mol. Life Sci. 70, 3171-3186 (2013)

Supplementary Information is available in the online version of the paper.

Acknowledgements We thank C. Benassayag, E. Farge, Y. Gachet, T. Lecuit, P.-F. Lenne, F. Payre, E. Sanchez-Herrero, B. Sanson and S. Tournier for comments on the manuscript, T. de Paula Peixoto for providing the graph-tool library, D. Kiehart, H. Steller, X. Wang, R. Ward, BDSC and DSHB for stocks and reagents, the TRI platform for imaging facilities and M. Aguirrebengoa for helping us with statistics. The Suzanne laboratory is supported by grants from the Agence Nationale de la Recherche (ANR), Fondation de la Recherche et de l'Innovation Thérapeutique en Cancérologie (RITC) and the University of Toulouse.

Author Contributions M.G., B.M. and M.S. have done the experiments in flies with the help of S.S. and A.G.; G.G. designed the simulation model. T.M. set up cell shape extraction, statistics and analysis in Matlab and performed the laser ablation experiments with the help of B.M.; M.S. supervised the project.

Author Information The source code for the model is released under the GNU General Public Licence and is available on GitHub (https://github.com/glyg/leg-joint and http://dx.doi.org/10.5281/zenodo.13386). Reprints and permissions information is available at www.nature.com/reprints. The authors declare no competing financial interests. Readers are welcome to comment on the online version of the paper. Correspondence and requests for materials should be addressed to M.S. (magali.suzanne@univ-tlse3.fr) for biology and materials and to G.G. (guillaume@damcb.com) for modelling. 


\section{METHODS}

Fly stocks and genetics. The fluorescent reporters used are the following: E-Cad$\mathrm{KI}(\mathrm{GFP})^{19}$, ubi::E-Cad::GFP ${ }^{20}$, uas::alpha-catenin-TagRFP ${ }^{21}$, uas::SCAT3 (FRET reporter of caspases activity ${ }^{22}$ ), sqh[AX3];;sqh::sqhGFP [40] and sqh[AX3];sqh:: sqhGFP [42] (MRLC::GFP) ${ }^{23}$, w;ap::Gal4,arm::arm-GFP;uas::mCD8-Cherry (generated using Bloomington stocks), w,Dlg1-GFP (CC01936, from Flytrap), Dll::Gal4 MD23, zfh2 LP30::Gal4 and UAS::Diap1 are described in Flybase, UAS::p35 (insertions in chromosome II and III) come from Bloomington.

Stocks for ectopic cell death induction are y,w,HS::flp;act $>y+>$ Gal4,uas::GFP and w;ptc::Gal4, uas::GFP; tub::Gal80ts (gifts from C. Benassayag), y,w,HS::flp; act5C $>\mathrm{CD} 2>\mathrm{Gal} 4$ (generated using Bloomington stocks), w; uas::lifeactGFP; uas: reaper (gifts from X. Wang and from Flybase),w; uas::hid(4),uas::DN-zip::GFP (gifts from H. Steller and D. Kiehart), w; uas::DN-zip::GFP; uas::rpr. Briefly, the progeny of crosses of interest were grown on standard medium at $25^{\circ} \mathrm{C}$. Third instar larvae were heat shocked for $15-20 \mathrm{~min}$ at $38^{\circ} \mathrm{C}$ and transferred to $29^{\circ} \mathrm{C}$ for $5.5 \mathrm{~h}-6 \mathrm{~h}$ before dissection. Following this treatment, a reproducible bias was observed, with clones essentially following the dorsal-ventral boundary in half of the wing pouch. When using ptc::Gal4 to induce cell death, crosses were performed at $18{ }^{\circ} \mathrm{C}$, the progeny was transferred at least $5.5 \mathrm{~h}$ to $30^{\circ} \mathrm{C}$ before dissection.

Immunostainings. Primary antibodies obtained from Developmental Studies Hybridoma Bank were: rat anti-E-Cad (DCAD2, 1:50), mouse anti-Arm (N2 7A1, 1:5) and mouse anti-Dlg (4F3, 1:200). Rabbit anti-cleaved Caspase 3 (9661, 1:100) and anti-cleaved Dcp1 (9578, 1:200) were obtained from Cell Signaling Technologies and chicken anti-beta-Gal (GTX77365, 1:1,000) was obtained from GeneTex. Mouse anti-Sqh (MRLC) and Guinea pig anti-Sqh1P $(1: 1,000)$ were gifts from R. Ward. Staining of the actin cytoskeleton was achieved using phalloidinRhodamine (1:200, Invitrogen) or phalloidin-Alexa647 (1:100, Interchim). Secondary antibodies coupled to Alexa- 488 or-555 were obtained from Fisher Scientific and diluted 1:200 while secondary antibodies coupled to Cy5 were obtained from Jackson Laboratories and diluted 1:50. Briefly, for immunostainings, imaginal tissues were fixed using paraformaldehyde (PFA) $4 \%$ diluted in PBS $1 \times$. Samples were washed and saturated using PBS $1 \times$, Triton X-100, 0.3\% BSA 1\% (BBT). Primary antibodies were diluted in BBT and incubated overnight at $4{ }^{\circ} \mathrm{C}$. Next, samples were washed and saturated in BBT, incubated with secondary antibodies (and phalloidin if required to stain F-actin) as indicated above, and subsequently washed with PBS $1 \times$, Triton X-100, $0.3 \%$. Samples were mounted in Vectashield containing DAPI (Vectors laboratories) and analysed under a Zeiss LSM710 laser scanning microscope. A similar protocol was followed for immunostainings on embryos except that fixation was performed for $5 \mathrm{~min}$ in heptane:formaldehyde 37\% (1:1). For E-cadherin and MyoII, embryos were devitellinised manually and stained immediately. Note that in order to preserve wing morphology in ectopic cell death experiments; dissections were performed in Schneider medium, followed by fixation with $4 \%$ PFA diluted in Schneider medium.

Time-lapse imaging. Leg discs were dissected in Schneider's insect medium (from Sigma Aldrich) supplemented with 2\% FCS and $0.5 \%$ penicillin-streptomycin. Ecdysone (from Sigma aldrich, 20-hydroxyecdysone H5142) was stored in a stock solution of $200 \mu \mathrm{g} \mathrm{ml}^{-1}$ at $-20^{\circ} \mathrm{C}$ and added to meet a final concentration of $2 \mu \mathrm{g} \mathrm{ml}^{-1}$. For in vivo imaging, leg discs were transferred on a coverslip in $15 \mu \mathrm{l}$ of the above medium complemented with methyl cellulose (from Sigma-Aldrich) at a final concentration of $2.5 \%$ to obtain a more viscous medium ${ }^{24}$. Spacers (Secure -Seal Imaging Spacers 0.12 depth from Sigma-Aldrich) were added between the coverslip and an air-permeable membrane (Lumox 25 from Sarstedt,) to avoid compression of the tissue, and halocarbon oil was added on the sides of the spacer to protect from dehydration. Note that forceps and scissors, as well as air-permeable membrane, were washed with ethanol before dissection. The membrane is rinsed with water and dried before use.

Before imaging, dissected leg discs may be incubated for 30 min with acridine orange (final concentration $0.1 \mu \mathrm{g} \mathrm{ml}^{-1}$ ) to reveal dying cells or with the red fluorescent lipid-binding dye FM4-64 (final concentration $36 \mu \mathrm{M}$ ). FM4-64 was also added at a concentration of $18 \mu \mathrm{M}$ in the imaging medium.

Imaging was essentially performed under an inverted laser scanning LSM710 confocal (Zeiss). Under our conditions, ex vivo leg development reproduces morphologic stages of pupal leg development characterized on fixed tissues (including leg evagination, local folding and the pattern of cell death), albeit at a lower speed (around 2 times).

Importantly, we found that image stacks of $30-40 \mu \mathrm{m}$ have to be taken every 3 min with optimal sectioning $(0.438 \mu \mathrm{m}$ for a $40 \times$ objective with 1.3 aperture and the pinhole set to $1 \mathrm{AU}$ ) in order to spot apico-basal myosin II cables and adhesion peaks as they are very dynamic structures.

Note that apoptotic cells were identified using the caspase FRET sensor (SCAT3 ${ }^{22}$, see section 'Post-imaging analysis' for details), acridine orange ${ }^{25}$ or FM4-64 that strongly labels apoptotic bodies.
Ex vivo culture and drug treatment. Q-VD-OPh is a broad spectrum caspase inhibitor which binds to the active site of activated proteases, more efficiently than the commonly used Z-VAD caspase inhibitor and is described as non-toxic even at extremely high concentrations ${ }^{26}$. Discs from white pupae (WP) were dissected in complemented Schneider medium as described above and incubated from pre-fold to mid-fold stage with either Q-VD-OPh (from R\&D systems; final concentration $500 \mu \mathrm{M}$ ) or DMSO (from Sigma Aldrich, final concentration 0.5\% in Fig. 2e and $0.06 \%$ in other experiments) in Fig. $2 \mathrm{c}$ (incubation of $6 \mathrm{~h}$ ) or Fig. 2e and Extended Data Fig. 4d (incubation of $4 \mathrm{~h}$ ). Q-VD-OPh was stored in a stock solution of $10 \mathrm{mM}$ in either DMSO 100\% (Fig. 2e) or in Schneider $+1.25 \%$ DMSO (other experiments). Throughout this study, we focused on the $\mathrm{t} 4 \mathrm{-t} 5$ fold, the only fold that is exclusively formed during the pupal stage and for which progression can be easily followed due to disc evagination. At WP stage, formation of the t4-t5 fold is not initiated yet, while other tarsal folds are partially formed. Hence, in this particular context of apoptosis inhibition, we observe, as expected, a slight perturbation of the formation of $\mathrm{t} 3-\mathrm{t} 4$ and strongly perturb the $\mathrm{t} 4-\mathrm{t} 5$ fold.

Post imaging analysis. SCAT3 FRET probe was previously described ${ }^{22}$. It is an indicator of caspase-3 activation using fluorescence resonance energy transfer (FRET) between an enhanced cyan fluorescent protein (the donor) and an enhanced yellow fluorescent protein (the acceptor) separated by a caspase cleavage site. FRET images of live leg discs were acquired with a Zeiss LSM710 microscope. A $458 \mathrm{~nm}$ laser was used to excite the sample. Cyan fluorescent protein (CFP) and yellow fluorescent protein (YFP) emission signals were collected through channel I (470$510 \mathrm{~nm}$ ) and channel II (525-600 nm), respectively. CFP and YFP images were acquired simultaneously. Sequential acquisition of CFP and YFP channels in alternative orders were tested and gave the same result as simultaneous acquisition. CFP and YFP images were processed by ImageJ software. A background region of interest was subtracted from the original image. Gaussian smooth filter was then applied to both channels. The final ratio image (YFP/CFP signal) was generated in ImageJ program.

Zen software (Zeiss) was used to generate three-dimensional reconstruction and sagittal views of tissues. Images were processed in Adobe Photoshop CS5 or ImageJ. Automated image analysis and quantifications of cell shape changes. Matlab, DipImage, Cell profiler and ICY were used to make automatic segmentation for quantification of cell shape in Fig. 2e and Extended Data Fig. 4d). Three-dimensional median filter was performed from confocal images before segmentation. A $Z$ projection based on the maximum intensity from each $Z$ stack was applied. An adaptive threshold was made to the $Z$ projection images to define the outline of cells based on the Otsu method. The local maximum of the "distance" function was used to find the centre of each cell to produce a resulting binary image. The distance transform is then used on the binary image to calculate the distance from cell membrane. The local maxima of the distance transform was calculated and were selected as seeds. For cells with high anisotropy the calculation of local maximum induced some errors and was manually corrected. The coordinate of each local maximum was used like seed to use a watershed algorithm function in Matlab and obtain a label matrix allowing the quantification of each cell object. The cells situated out of the domain of interest were rejected. To perform statistics we calculated the maximum and the minimum vertical coordinates based on the coordinates of the centre of outlined cells. Then 12 domains were defined based on this coordinate. The calculation of anisotropy was defined as the ratio between the lengths of the principal axes of an ellipsis fitting the cell contour.

Measurements of actomyosin levels. To quantify junctional acto-myosin (Extended Data Fig. 3c). For each leg disc, acto-myosin intensity was measured using Image) by drawing a circular region of interest of $1 \mu \mathrm{m}^{2}$ in 8 individual junctions in the fold domain (t4-t5) and in 8 individual junctions in the segment domain ( $\mathrm{t} 4)$. The measurements done in the fold were then normalized with the mean value of actomyosin intensity in the segment domain independently in each leg disc (to insure the presence of an internal control). As a consequence, the values represent the differential accumulation of junctional acto-myosin in the fold compared to the segment domain

To quantify acto-myosin per surface unit (Extended Data Fig. 3d, e, g). For each leg disc, acto-myosin intensity was measured using ImageJ by drawing a segmented line either in the fold ( $t 4-\mathrm{t} 5)$ or in the segment $(\mathrm{t} 4)$ domain. These values were divided by the length of the line and each measure in the fold was then normalized by the corresponding segment domain value (to insure the presence of an internal control). As a consequence, the values represent the differential accumulation per surface unit of acto-myosin in the fold compared to the segment.

Statistics. To calculate $P$-values, we used the non-parametric Wilcoxon rank sum test (also called Mann and Whitney test) since samples do not follow a normal distribution and do not have equal variances in Extended Data Fig. 3c-e, g, Extended Data Fig. 4d and Extended Data Fig. 9d. The null hypothesis is that the measures are samples from continuous distributions with equal medians. The test considers that the samples are independent and in Extended Data Fig. 4d, it takes into account 
that the control and Q-VD-OPh can have different lengths. For cell shape characteristics (anisotropy, area and orientation), values are represented by box plot (the red line represent the median). For acto-myosin level quantifications, values are represented as mean values with standard error bars.

Photo-ablation experiments. Laser-ablation experiments in prepupal leg imaginal discs were performed with a pulsed Q-switched microchip double-frequency Nd: YAGlaser ( $\lambda=532 \mathrm{~nm}, 550 \mathrm{ps}, 7 \mathrm{kHz}, 3.5 \mu \mathrm{J}$ per pulse). The laser beam was focused through a high numerical aperture oil-immersion lens $(\times 60 \mathrm{C}$-Apochromat NA 1.4 , Leica) to produce an experimental beam waist around $600 \mathrm{~nm}$ in the focal plane. Photo-disruption was produced in the focal plane due to the plasma generation in the middle of the adherens junctions after a total of 21 laser pulses of $3 \mathrm{kHz}$. A pair of Galvanometric mirrors were conjugated to the black focal plane of the microscope objective lens to stir the ablation beam and allow simultaneous ablation of two junctions. We used an inverted microscope (Leica DMI6000B) with wide field illumination to produce fluorescent live imaging of adherens junctions labelled with arm::GFP fusion protein. Adherens junctions were placed in the centre of the field to produce a better reproducibility. METAMORPH coupled to ILAS software controlled the laser and the microscope (RopperScientifiC SA). The Images were taken every second over a period of $30 \mathrm{~s}$. Note that for laser ablations, in absence of cell death (incubation with Q-VD-OPh, Extended Data Fig. 3i), expression of a membrane-bound cherry protein under the control of the apterous driver (w;ap::Gal4, arm::arm-GFP; uas::mCD8-Cherry; for expression pattern, see Extended Data Fig. 4c) was used to visualize the fold domain since cell shape changes do not occur in these conditions.

Modelling. Our apical junction network model is a generalization to threedimensional of the Farhadifar et al. model ${ }^{15}$. In order to allow non-planar interactions, the surface elasticity is replaced by a volume elasticity. The apico-basal interaction is modelled as an energy proportional to cell height, with the same linear form as the apical line tension. The initial epithelium is represented as an hexagonal lattice of cells over a cylinder. Next, each cell follows a division process with a random division plane orientation. The order of cell divisions on the epithelium is chosen randomly, uniformly across the epithelium. Division is modelled by first increasing the cell equilibrium volume, finding the local energy minimum and second by dividing the cell and finding the new local energy minimum. Then, apoptotic cells are chosen randomly in a region around the centre of the epithelium with a biased distribution reproducing the in vivo cell death pattern. Apoptosis is performed by gradually diminishing the cell preferred volume, increasing its contractility and rising the apico-basal tension term (ten steps are performed). At each step, the local energy is minimised by a gradient descent strategy. After the first step of the first apoptotic cell is performed, the second cell starts its apoptosis, and so on. Once ten steps are performed for one cell, it is removed from the tissue, such that no centripetal force remains at that point. When removing the apoptotic cell, its neighbours are rearranged through a series of type 1 intercalations (see Faradifar et al. ${ }^{15}$ ). To model acto-myosin activity increase in the neighbouring cells, contractility is increased in the neighbouring cells by a factor the amplitude of which decreases exponentially with the distance to the apoptotic cells. After a cell has been eliminated and its neighbours rearranged, the whole tissue is brought back to equilibrium by successively computing the local energy minimum for all the cells of the tissue in a random order. Technically, the epithelium is described as an oriented graph in the graph-tool library ${ }^{28}$ (http://graph-tool.skewed.de) in the Python programming language. Energy minimisation is performed with the scipy library ${ }^{29}$ (http://scipy.org), using the Broyden, Fletcher, Goldfarb, and Shanno bound constrained minimisation algorithm provided by this library. The source code for the model is released under the GNU General Public Licence and is available on github at https:/github.com/glyg/leg-joint and http://dx.doi.org/10.5281/zenodo.13386. Extensive details on the numerical method as well as a complete derivation of the gradient can be found at this address.

The apical contractility and linear tension parameters $\Gamma$ and $\Lambda$ where chosen so that the relation between cell apical area and number of neighbours follows the same linear increase as observed by Farhadifar et al..$^{15}$ in the wing disc (compare Fig. $2 \mathrm{~g}$ of Farhadifar et al. ${ }^{15}$ with Extended Data Fig. $5 \mathrm{~b}$ of the present work). We explored a range of apoptotic cell number, apico-basal force amplitude and contractility increase values (Extended Data Figs 6,7,8) and observed that cell morphology in the fold is best reproduced with amplitudes of $1 \Gamma$ and $1 \Lambda$. The fact that they are in the same order of magnitude as the apical interactions gives us confidence that the chosen parameters are realistic. Furthermore, our tissue has a consistent morphology with or without apoptosis, increased apical contractility or apico-basal force, meaning that the balance of forces is coherent and equilibrated.

For cell shape analysis, a sector centred $30^{\circ}$ above the ventral most part of the cylindrical epithelium was extracted. Radius and area were normalized with respect to their average values on the simulated epithelium in the absence of apoptosis.

19. Huang, J., Zhou, W., Dong, W., Watson, A. M. \& Hong, Y. From the Cover: Directed, efficient, and versatile modifications of the Drosophila genome by genomic engineering. Proc. Natl Acad. Sci. USA 106, 8284-8289 (2009).

20. Oda, H. \& Tsukita, S. Real-time imaging of cell-cell adherens junctions reveals that Drosophila mesoderm invagination begins with two phases of apical constriction of cells. J. Cell Sci. 114, 493-501 (2001).

21. Ishihara, S. \& Sugimura, K. Bayesian inference of force dynamics during morphogenesis. J. Theor. Biol. 313, 201-211 (2012).

22. Takemoto, K., Nagai, T., Miyawaki, A. \& Miura, M. Spatio-temporal activation of caspase revealed by indicator that is insensitive to environmental effects. J. Cell Biol. 160, 235-243 (2003).

23. Royou, A., Sullivan, W. \& Karess, R. Cortical recruitment of nonmuscle myosin II in early syncytial Drosophila embryos: its role in nuclear axial expansion and its regulation by Cdc2 activity. J. Cell Biol. 158, 127-137 (2002).

24. Aldaz, S., Escudero, L. M. \& Freeman, M. Live imaging of Drosophila imaginal disc development. Proc. Natl Acad. Sci. USA 107, 14217-14222 (2010).

25. Abrams, J. M., White, K., Fessler, L. I. \& Steller, H. Programmed cell death during Drosophila embryogenesis. Development 117, 29-43 (1993).

26. Caserta, T. M., Smith, A. N., Gultice, A. D., Reedy, M. A. \& Brown, T. L. Q-VD-OPh, a broad spectrum caspase inhibitor with potent antiapoptotic properties. Apoptosis 8, 345-352 (2003)

27. Guarner, A. et al. The zinc finger homeodomain-2 gene of Drosophila controls Notch targets and regulates apoptosis in the tarsal segments. Dev. Biol. 385, 350-365 (2014).

28. Peixoto, P. T. The graph-tool python library. figshare http://dx.doi.org/10.6084/ m9.figshare.1164194 (2014).

29. van der Walt, S., Colbert, S. C. \& Varoquaux, G. The NumPy Array: a structure for efficient numerical computation. Comput. Sci. Eng. 13, 22-30 (2011). 
a

Dlg::GFP

Acridine Orange

$20 \mu \mathrm{m}$
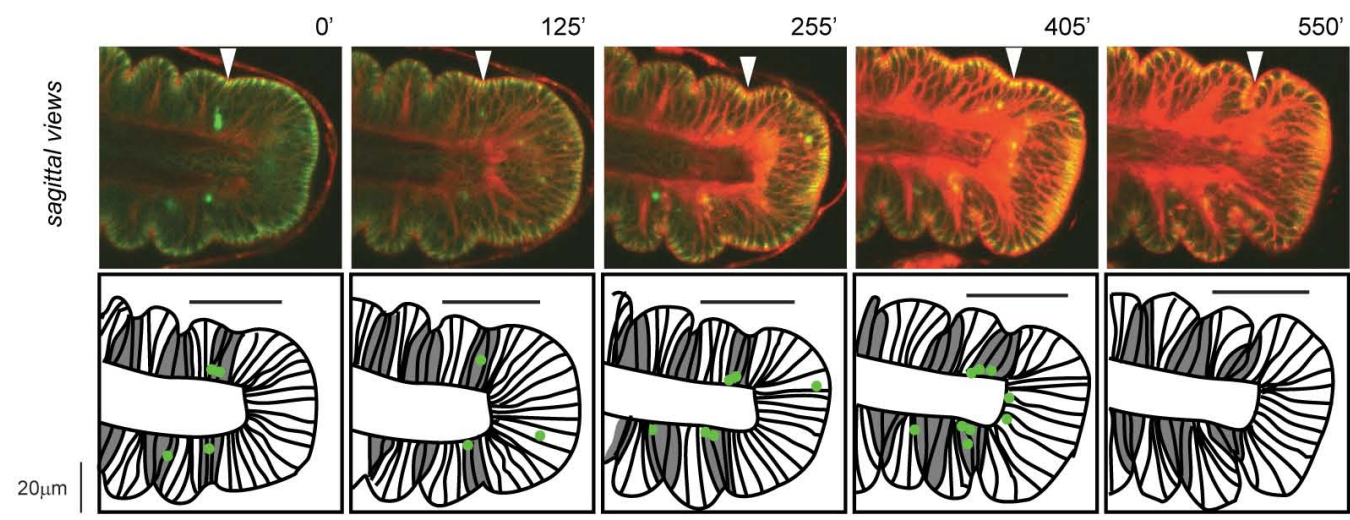

b
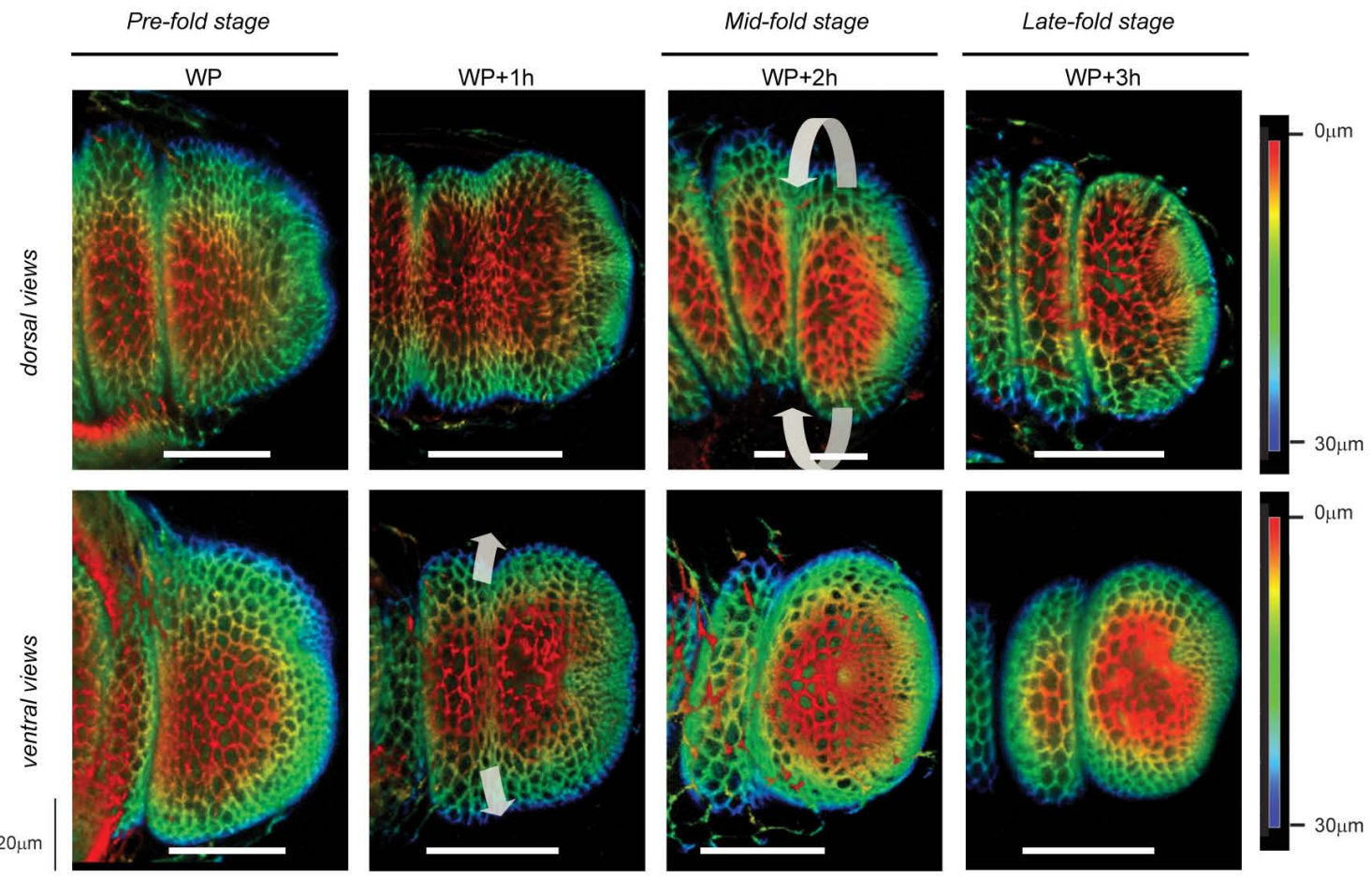

C

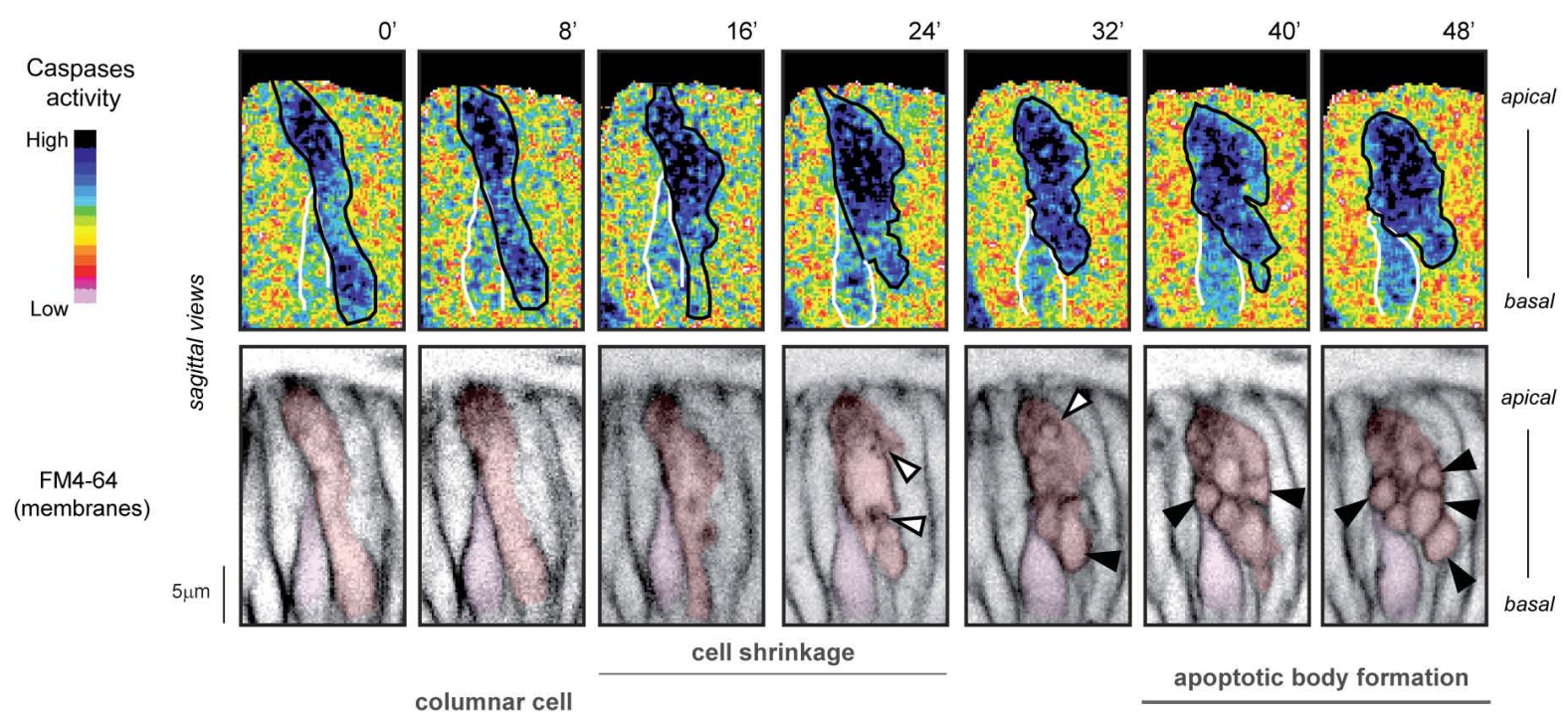

membrane blebbing 
Extended Data Figure $1 \mid$ Spatio-temporal pattern of fold formation and hallmarks of apoptosis. This figure is associated with Fig. 1. a, Time-lapse images and schematics of the distal region of a Dlg::GFP leg disc from pre-fold stage (WP) showing the progression of the $\mathrm{t} 4$ - $\mathrm{t} 5$ fold (white arrowheads). Acridine orange was used to stain dying cells (green) and FM4-64 to stain membranes (red). Note the presence of apoptotic cells in the fold region (coloured in grey in the schematics below, $n=27$ ). The t4-t5 domain is indicated by a black line on the schematics. $\mathbf{b}$, Three-dimensional reconstruction of the distal region of Dlg::GFP pupal legs undergoing fold morphogenesis. The colour code indicates tissue depth. Images show legs at different stages of development, from WP to WP $+3 \mathrm{~h}$. Throughout this study, we have focused on the $\mathrm{t} 4$ - $\mathrm{t} 5$ fold, the only one that is exclusively formed during pupal stage and for which progression can be easily followed due to disc evagination. For each time point, top and bottom panels show dorsal and ventral views of leg discs respectively, and the t4-t5 domain is indicated by a white line. Note that the fold is initiated in the most ventral part of the leg, then progresses laterally (arrows) to end in the most dorsal part of the leg ( $n=10$ for WP, $n=7$ for WP $+1 \mathrm{~h}, n=10$ for WP $+2 \mathrm{~h}, n=10$ for $\mathrm{WP}+3 \mathrm{~h}$ ). c, High magnification images from a time-lapse video during apoptosis showing caspase activity, revealed by the FRET construct SCAT3 (top), and the outline of cell membranes, revealed by FM4-64 staining (bottom) $(n=19)$. These images illustrate that the classical apoptotic stages, including shrinkage, blebbing (hollow arrowheads) and fragmentation (black arrowheads), are recapitulated in the developing Drosophila leg epithelium. Black outline (top) and red false-colour (bottom) highlight the apoptotic cell. Another apoptotic cell (outlined in white and coloured in pink) has also just turned on the apoptotic pathway. Note that in both cases the apoptotic pathway is turned on before visible morphological change. 
a

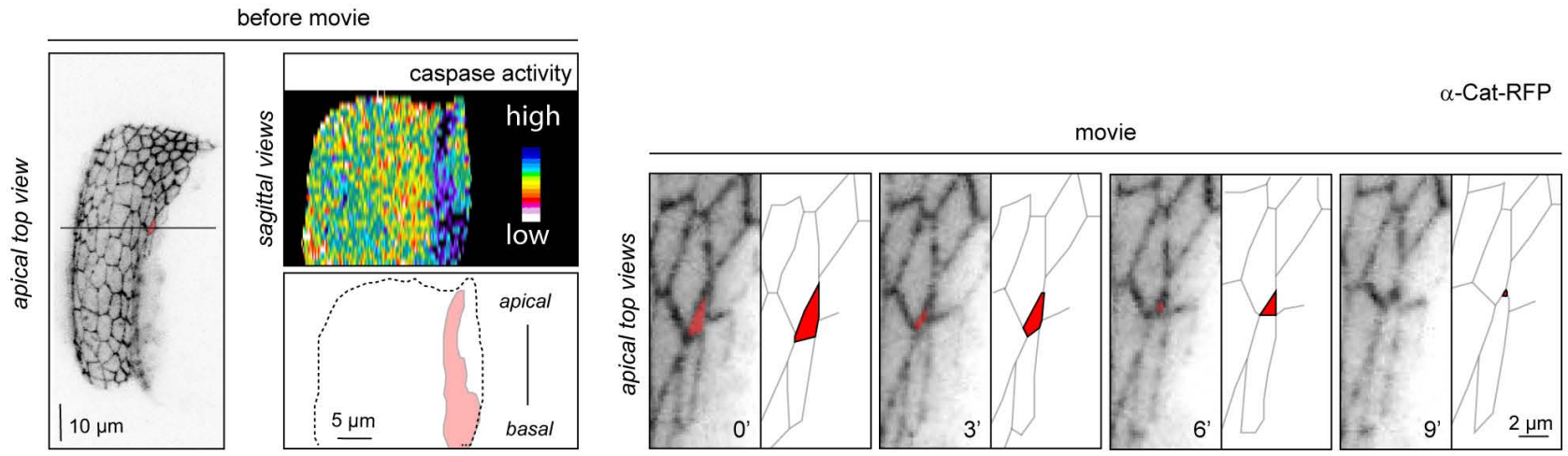

b

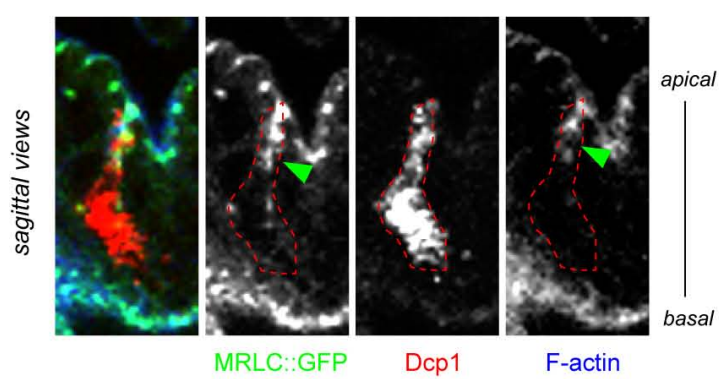

d

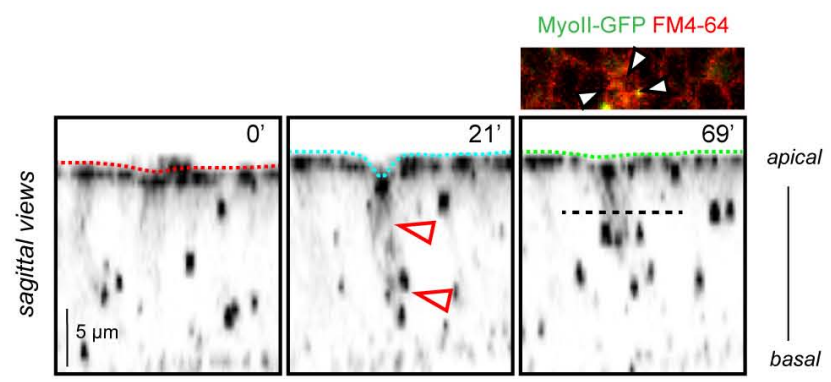

MRLC::GFP

h
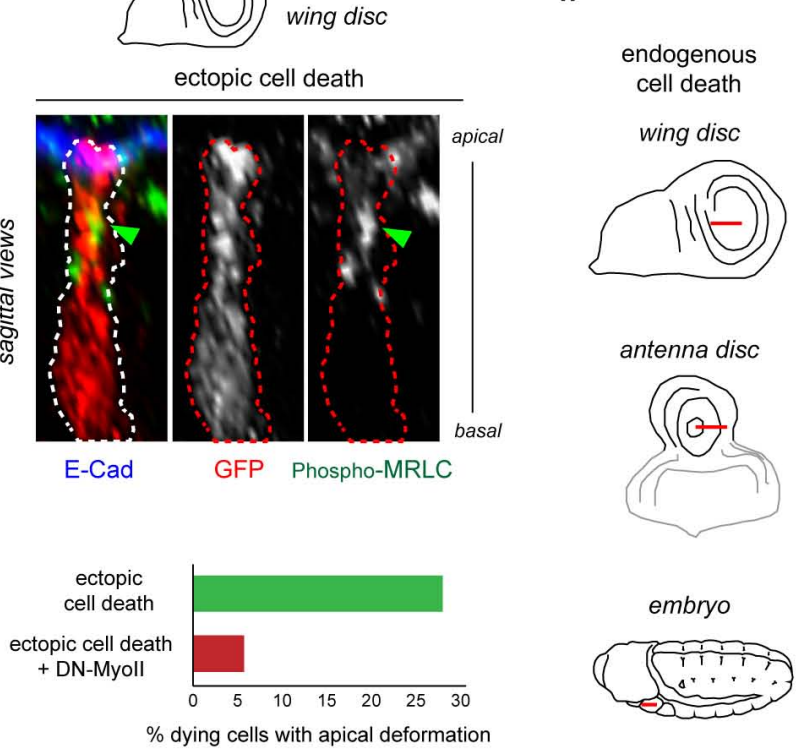

c

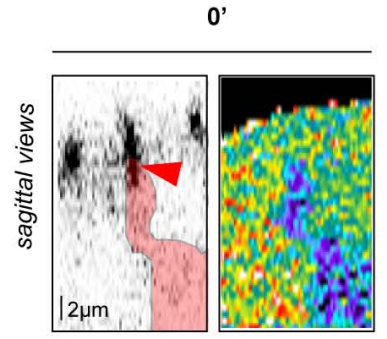

$\alpha$-Cat-RFP caspase activity

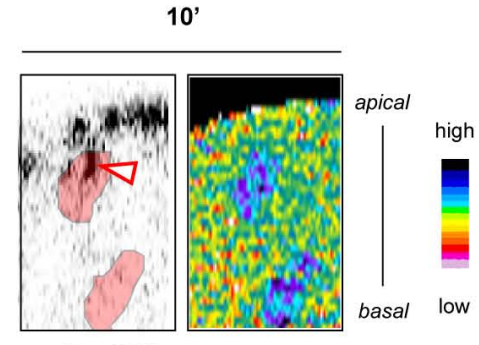

$\alpha$-Cat-RFP caspase activity

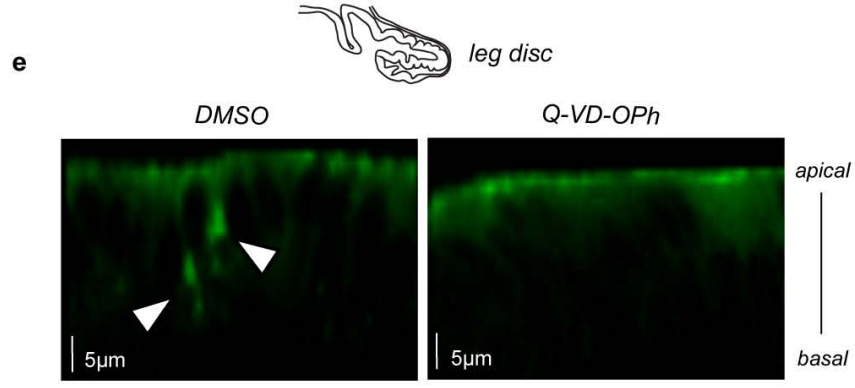

MRLC:.GFP

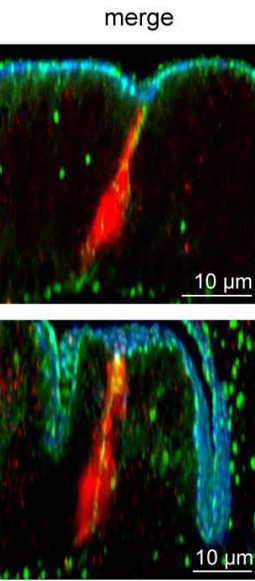

Cleaved Caspase

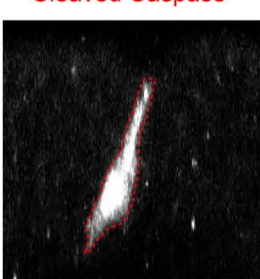

MRLC::GFP
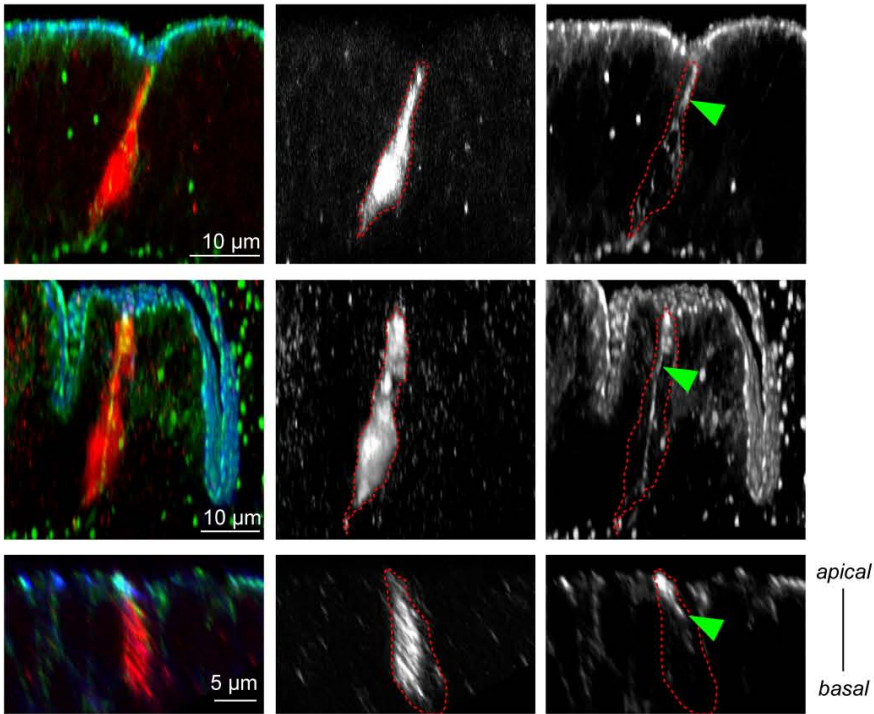
Extended Data Figure $2 \mid$ Adherens junctions and acto-myosin cytoskeleton dynamics in apoptotic cells. This figure is associated with Fig. 1. a, Time lapse images of a pupal leg disc expressing $\alpha$ Cat-TagRFP in the ap domain (which encompasses the 4 - $\mathrm{t} 5$ fold) and the FRET construct SCAT3 to reveal caspase activity and thus visualize apoptotic cells $(n=10)$. The apoptotic cell outline is visible on the sagittal section and represented on the scheme. The position of the sagittal section is indicated by a black line on the apical top view to the left. Note the reduction of the apical surface of the apoptotic cell (apical top views on the right). The apoptotic cell is highlighted in red on both panels. b, Acto-myosin cable (green arrowhead) observed in a single apoptotic cell from a pupal leg disc $(n=6)$. MRLC::GFP is in green, F-actin (stained with phalloidine) is blue and cleaved Dcp1 is red. c, Sagittal sections of an apoptotic cell (visualized with the FRET construct SCAT3) before and after fragmentation, illustrating the apoptotic adhesion peak $(n=27$, red arrowhead) and its splitting up (hollow red arrowhead) when the apoptotic cell detaches at fragmentation. d, Time-lapse images of a MRLC::GFP pupal leg disc showing apical surface release upon apoptotic cell fragmentation (timepoint $69 \mathrm{~min}, n=12$ ). Fragmentation is clearly visible in the $z$ section (top) showing membrane staining of the apoptotic fragments with FM4-64 (white arrowheads) (the position of the $z$ section is indicated by a black dotted line in the sagittal view). The myosin II cable is indicated by hollow open red arrowheads in sagittal sections. e, MRLC::GFP leg discs incubated in either
DMSO (control) or Q-VD-OPH (cell death inhibition), showing the absence of the apico-basal myosin II cable in the absence of cell death (right, $n=0$ out of 30). Arrowhead points out to the myosin II cable in the control $(n=14$ out of 30). f, A single apoptotic cell (same cell shown in Fig. 1f, identified by GFP expression, red) generated by ectopic expression of reaper in the wing disc (from y,w,hs::flp; act-frt-y+-frt-Gal4, uas::GFP / uas::lifeactGFP; uas:::rpr larvae). The green arrowhead points out to the myosin II cable stained by antiphospho-Sqh/MRLC (green, $n=11$ ). The dotted red line outlines the dying cell. g, Percentage of individual apoptotic cells (with or without myosin II activity) with apical deformation. This quantification is associated with Fig. If, $\mathrm{g}$ ( $n=68$ and $n=106$, respectively). Genotype for ectopic cell death is y,w,hs::flp; act-frt-stop-frt::Gal4, uas::GFP / uas:::lifeactGFP; uas::rpr and genotype for ectopic cell death without myosin II activity specifically in apoptotic cells is y,w,hs::flp; uas::DN-zip::GFP, uas::hid / act-frt-stop-frt::Gal4. Note that, in the latter condition, myosin II activity is maintained in the neighbouring cells. $\mathbf{h}$, Sagittal sections close-ups of MRLC::GFP wing and antennal discs from 3rd instar larvae and a MRLC::GFP stage 11 embryo showing that an apico-basal structure of myosin II (green arrowheads) is formed in dying cells in each of these different tissues $(n=6,7$ and 7 , respectively). Cleaved Dcp1 (activated caspase) is in red, E-Cad in blue (wing) and $\mathrm{Arm} / \beta \mathrm{Cat}$ in blue. The region shown in each close up is indicated by a red line on schematics on the left. 
a

b
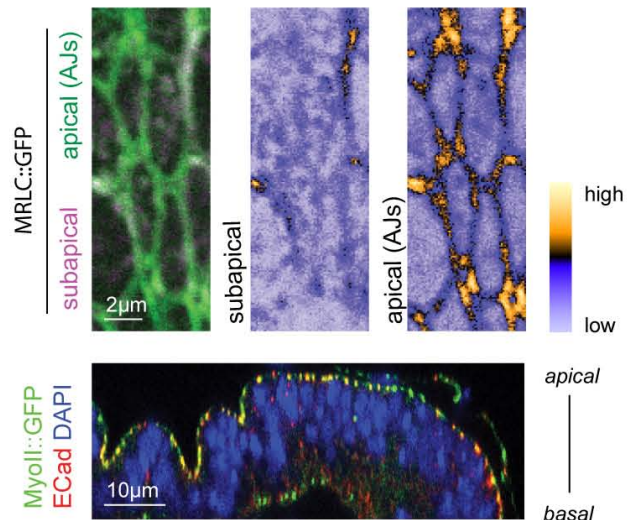

d
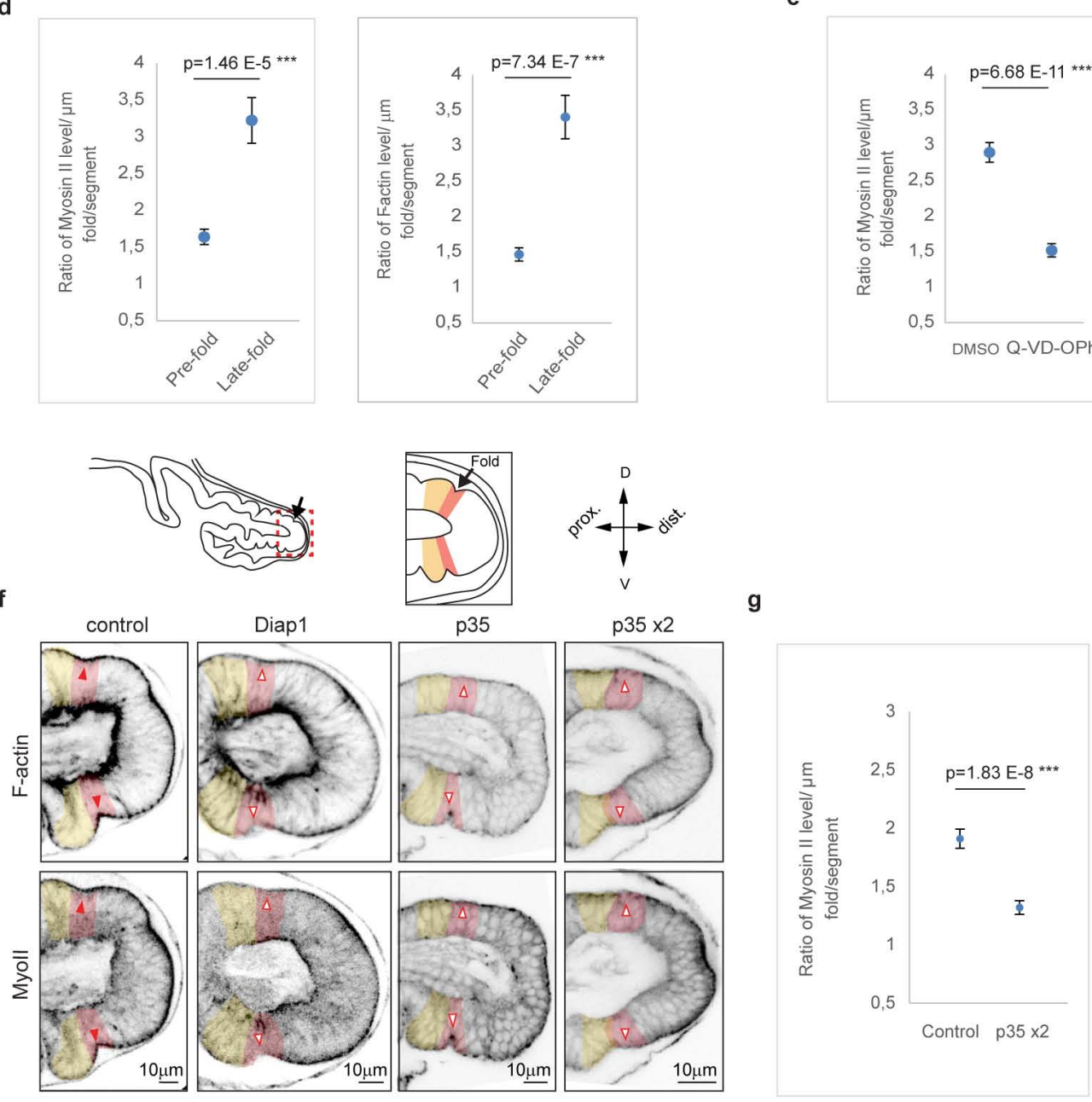
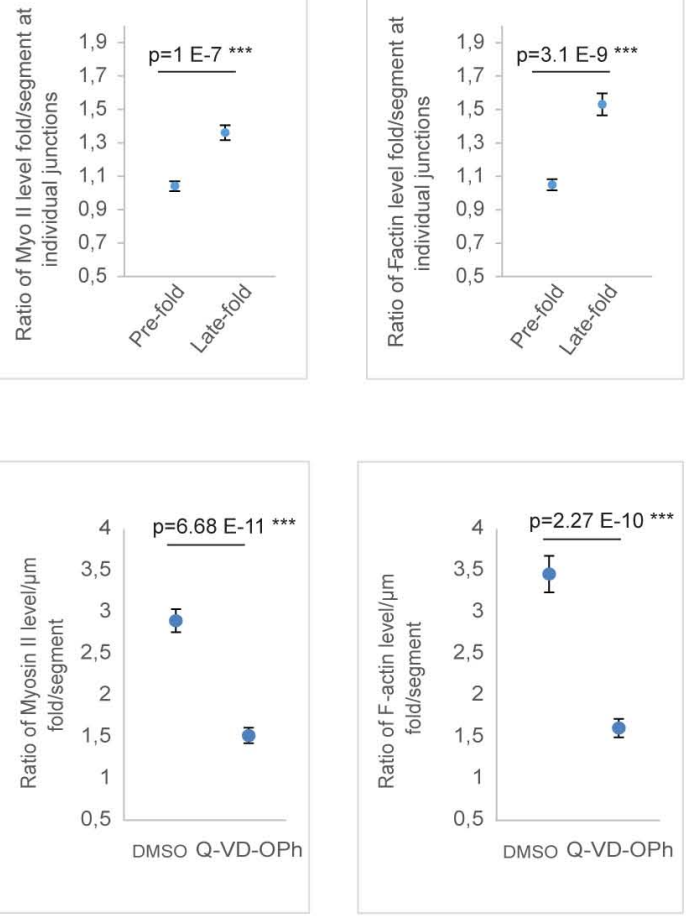

h

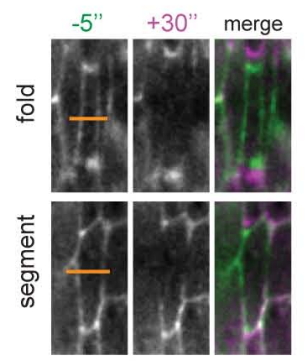

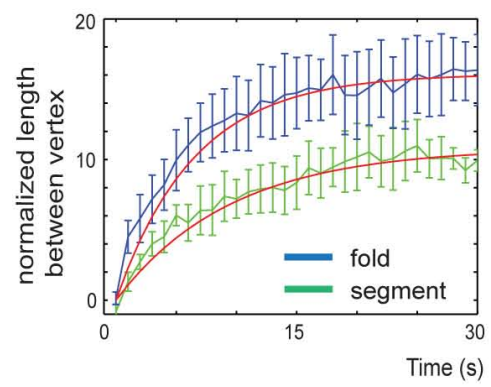
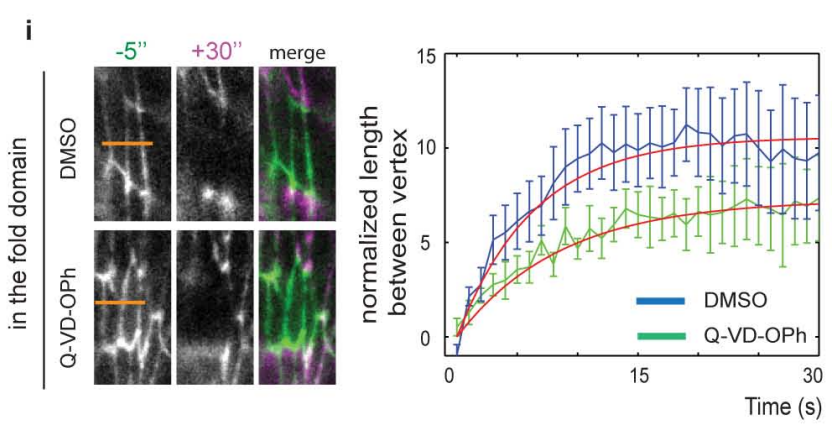
Extended Data Figure $3 \mid$ Myosin II and tension dynamics during fold formation. This figure is associated with Fig. 2. a, Close-up views of cells expressing MRLC::GFP, showing that, in the leg disc, myosin II is preferentially accumulated at the cortex (green) rather than at the medio-apical web (magenta). Relative intensity of myosin II at both levels is shown on separate panels $(n=22)$. b. Sagittal section of a leg disc from pre-fold stage (WP) showing that myosin II (visualized by MRLC::GFP construct, in green) colocalizes with E-Cad at adherens junctions (labelled in red) $(n=22)$. c, Quantification of myosin II::GFP (left) and F-actin (right) levels at individual junctions in fold (t4-t5) and segment ( $\mathrm{t} 4)$ domains of leg discs from pre-fold (young WP $+1 \mathrm{~h}$ ) and late-fold (young WP $+4 \mathrm{~h}$ ) stages $(n=144$ and $n=112$, respectively). Values are represented as mean values with error bars representing standard errors. The intensity of the signal in the fold has been normalized with the mean intensity in the segment domain. We used the non-parametric Wilcoxon rank sum test (also called Mann and Whitney test). d, e, Quantification of myosin II::GFP (left) and F-actin (right) levels per surface unit in fold (t4-t5) and segment (t4) domains of (d) leg discs from pre-fold (young $\mathrm{WP}+1 \mathrm{~h})$ and late-fold (young $\mathrm{WP}+4 \mathrm{~h})$ stages $(n=23$ and $n=18$ measurements respectively) or (e) in dissected discs cultured from pre-fold to mid-fold stage with either DMSO (control) or Q-VD-OPh (cell death inhibition) ( $n=32$ and $n=24$ measurements respectively). Values are represented as mean values with error bars representing standard errors. The intensity of the signal in the fold has been normalized with the mean intensity in the segment domain. We used the non-parametric Wilcoxon rank sum test (also called Mann and Whitney test). f, Sagittal sections of pupal leg discs from mid-fold stage (WP $+2 \mathrm{~h}$ ) of the following genotypes: $D l l-$ Gal4 ${ }^{[\mathrm{MD} 23]}$ (ctl, $n=8$ ), UAS-DIAP1;LP30-Gal4 (Diap1, $n=27$ ), Dll$\mathrm{Gal}^{[\mathrm{MD} 23]} /$ UAS-p35 (p35, $\left.n=11\right)$ and Dll-Gal4 ${ }^{[\mathrm{MD} 23]} /$ UAS-p35; UAS-p35 (p35 $\mathrm{x} 2, n=15$ ) (at early pupal stages, LP30-Gal4 and Dll::Gal4[MD23] show similar expression patterns, namely expression in the distal tibia and in all tarsal segment ${ }^{27}$ ). The stabilization of myosin II and F-actin in the t4-t5 fold observed in the control (red arrowheads) is reduced or absent when cell death is inhibited (open arrowheads point out to the $t 4-\mathrm{t} 5$ domain in the context of cell death inhibition). Myosin II is detected using anti-Sqh/MRLC antibody. The fold domain is false-coloured in pink and the segment domain in yellow. g, Quantification of myosin II (using anti-sqh antibody, left) and F-actin (right) levels per surface unit in fold (t4-t5) and segment (t4) domains in leg discs from mid-fold stage (WP $+2 \mathrm{~h})$ in control (DllGal4) and cell inhibition (Dll $>$ p $35 \times 2$ ) contexts ( $n=25$ and $n=28$ measurements respectively). Values are represented as mean values with error bars representing standard errors. The intensity of the signal in the fold has been normalized with the mean intensity in the segment domain. We used the non-parametric Wilcoxon rank sum test (also called Mann and Whitney test). $\mathbf{h}$, Laser ablation experiments of apical membranes in arm::GFP leg discs in the segment domain versus the fold domain where apoptosis takes place. Discs were dissected and cultured ex vivo from pre-fold stage (WP) to mid-fold stage. Note the increase in the length of vertex release in the fold domain compared to the segment domain $(n=4)$. i, Laser ablation experiments of apical membranes in the fold domain of arm::GFP leg discs incubated from pre-fold stage (WP) to mid-fold stage with either DMSO (control, $n=5$ ) of Q-VD-OPH (cell death inhibition, $n=4$ ). Note that vertex release in the fold is reduced in the absence of apoptosis. h, i, Right panels, graphs representing quantifications of the increase in distance between vertices following laser cut, revealing apoptosisdependent increased cellular tension in the fold domain versus the segment domain. Examples of ablated cells before (green) and after (magenta) laser cut are shown on the left. Orange bars represent the region where the laser cut has been performed. Errors bars correspond to the standard error of the mean. 
a

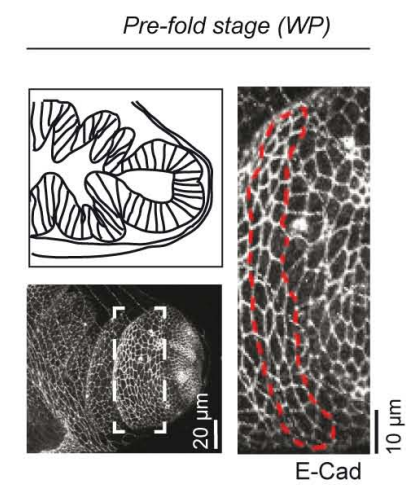

c

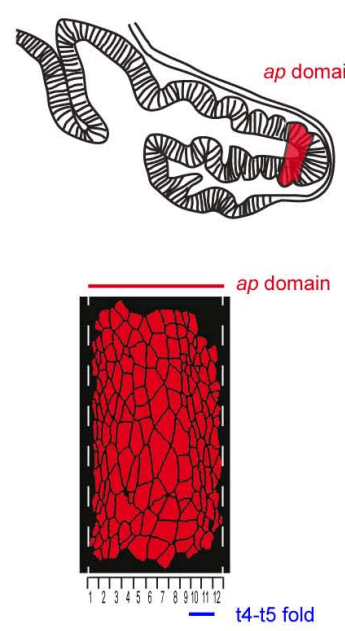

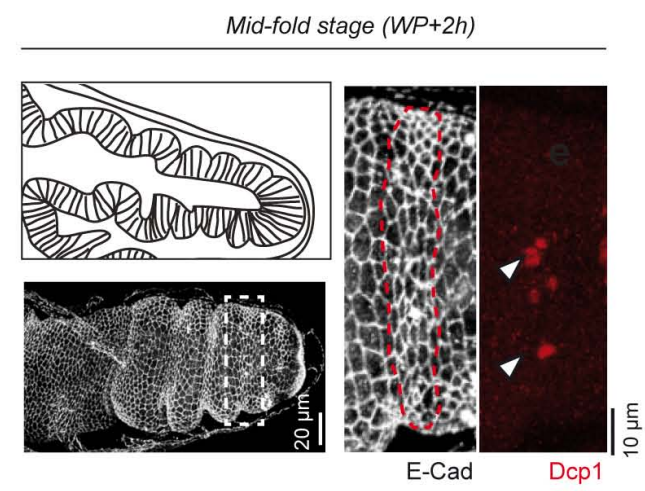

d b

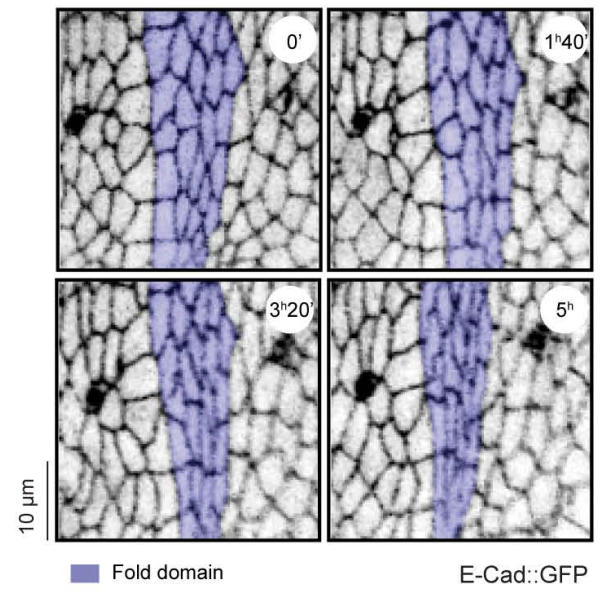

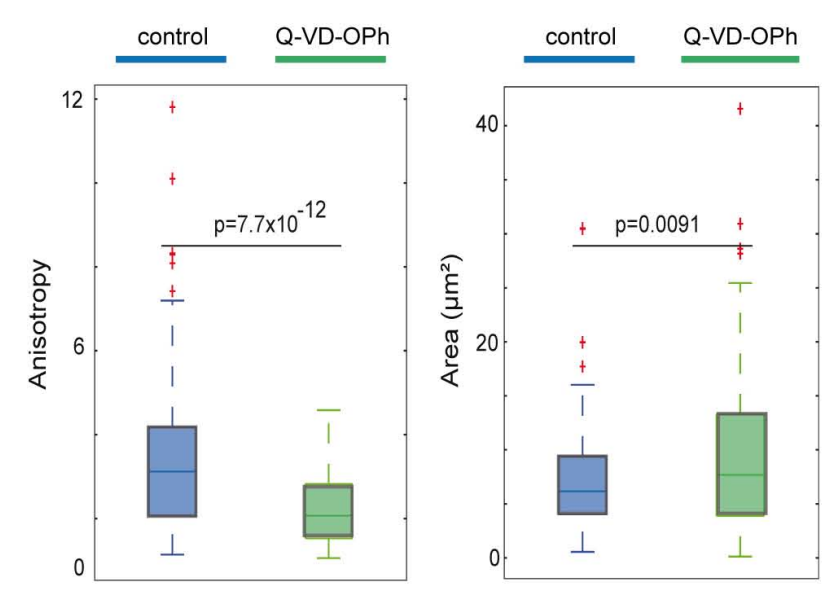
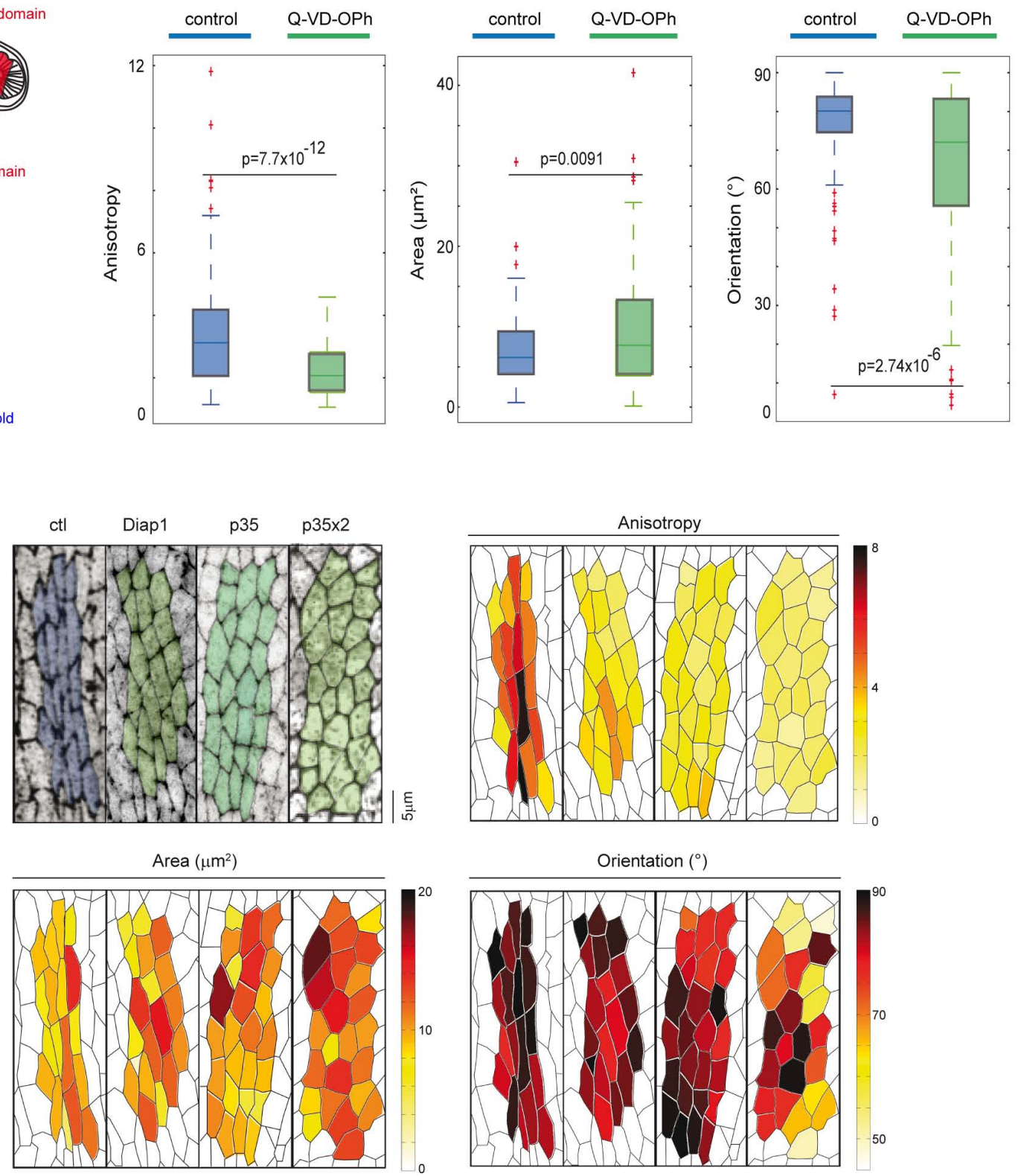
Extended Data Figure $4 \mid$ Cell shape dynamics during fold formation. This figure is associated with Fig. 2. a, Three-dimensional reconstructions and schematics of leg imaginal discs from pre-fold stage (WP) and mid-fold stage $(\mathrm{WP}+2 \mathrm{~h})$ stained with E-Cad in white and cleaved Dcp1 (revealing caspase activity) in red ( $n=11$ for each). High magnifications of the fold domain (surrounded in red) are shown on the right hand side of each panel.

Arrowheads indicate apoptotic cells. b, Cell shape dynamics during fold formation $(n=8)$. c, Schematic of a pupal leg disc showing the apterous domain (in red) in the $\mathrm{t} 4$ tarsal segment and overlapping the 4 - $\mathrm{t} 5$ fold (top). The bottom panel shows a typical result of automated cell outline extraction of a pupal leg disc double-stained for adherens junctions and the apterous domain. Cells from this domain have been subdivided into 12 sections along the proximo-distal axis (see Methods). d, Anisotropy, area and orientation of cells from DMSO (control, $n=7$ ) or Q-VD-OPH (cell death inhibition, $n=8$ ) leg discs from fold domain sections 10 and 11 (see Extended Data Fig. 4c) were quantified and values represented as box plot. Discs were dissected and incubated from pre-fold stage (WP) to mid-fold stage. We used the nonparametric Wilcoxon rank sum test (also called Mann and Whitney test). e, Three-dimensional reconstruction images of anti E-Cad stained pupal leg discs from mid-fold stage (WP $+2 \mathrm{~h}$ ) (top left) of the following genotypes: LP30::Gal4 (control, ctl, $n=11$ ), uas-DIAP1; LP30::Gal4 (Diap1, $n=22$ ), Dll::Gal4[MD23] / uas-p35 (p35, $n=9$ ) and Dll::Gal4[MD23] / uas-p35; uas-p35 (p35 x2, $n=8$ ) (at early pupal stages, LP30-Gal4 and Dll::Gal4[MD23] show similar expression patterns, namely expression in the distal tibia and in all tarsal segments ${ }^{27}$ ). For each condition, cell outlines were extracted and anisotropy, apical surface area and orientation of cells from the fold domain were quantified and colour-coded. Note that when cell death is inhibited, anisotropy is reduced, apical surfaces are increased and cell preferential orientation is perturbed compared to the control situation. 
a

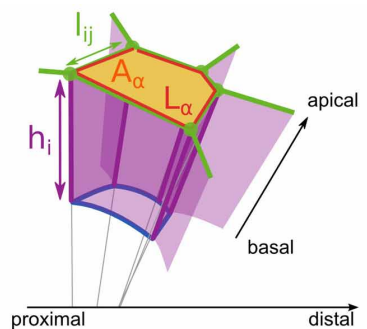

Force balance

at vertex $\mathrm{i}$

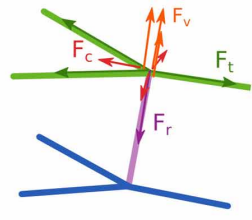

junction vertex junction edge

ij edge length

$h_{i}$ junction height

Kapical tension $\quad E_{t}=\mathrm{N}_{\mathrm{ij}}$

« apical contractility $\quad E_{\mathrm{c}}=\Gamma \mathrm{L}^{2}$

7) volume elasticity $E v=K_{v}\left(h_{a} A_{a}-V_{0}\right)^{2}$

$\downarrow$ apico-basal force $\quad E_{\mathrm{r}}=\mathrm{Rh}_{\mathrm{i}}$ b

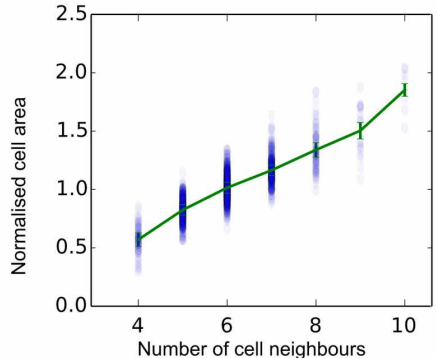

c

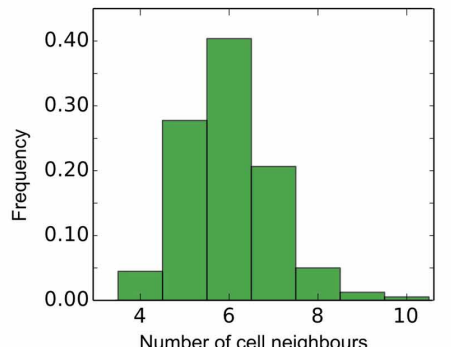

d

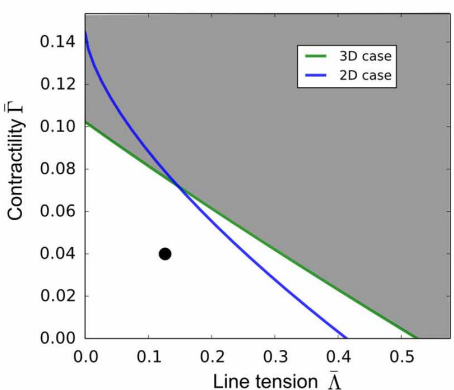

Extended Data Figure $5 \mid$ Three-dimensional epithelial cell model. This figure is associated with Fig. 3. a, Each cell is represented as an apical surface delimited by apical junctions. Each cell interacts with its neighbours through the apical junctions at its borders. In the original work by Farhadifar et al. ${ }^{15}$, three interactions are considered: (1) the tension opposing the elongation of a particular junction edge, with energy increasing with edge length; (2) a contractility, with energy proportional to the cell perimeter squared, used to model cell constriction; (3) a surface elasticity bringing the apical cell area back to a preferred area. As in the original work, the model can produce cell division, types one and three transitions, to which we added apoptosis. Yet in our case, contrary to Jüllicher and colleagues work ${ }^{15}$, we must also take into account non planar modifications of the epithelial sheet. To this end, we modified the elastic area interaction to take into account a constrain on cell volume. The new interaction is termed volume elasticity and transmits contractions and dilations of the apical sheet along the apical-basal axis. The associated energy is proportional to the square of the difference between the current cell volume and a preferred volume. $\mathbf{b}$, Average areas of cells as a function of the number of neighbouring cells in the epithelium before apoptosis, to be compared with Fig. $2 \mathrm{~g}$ in Farhadifar et al. ${ }^{15}$. Our tissue shows a

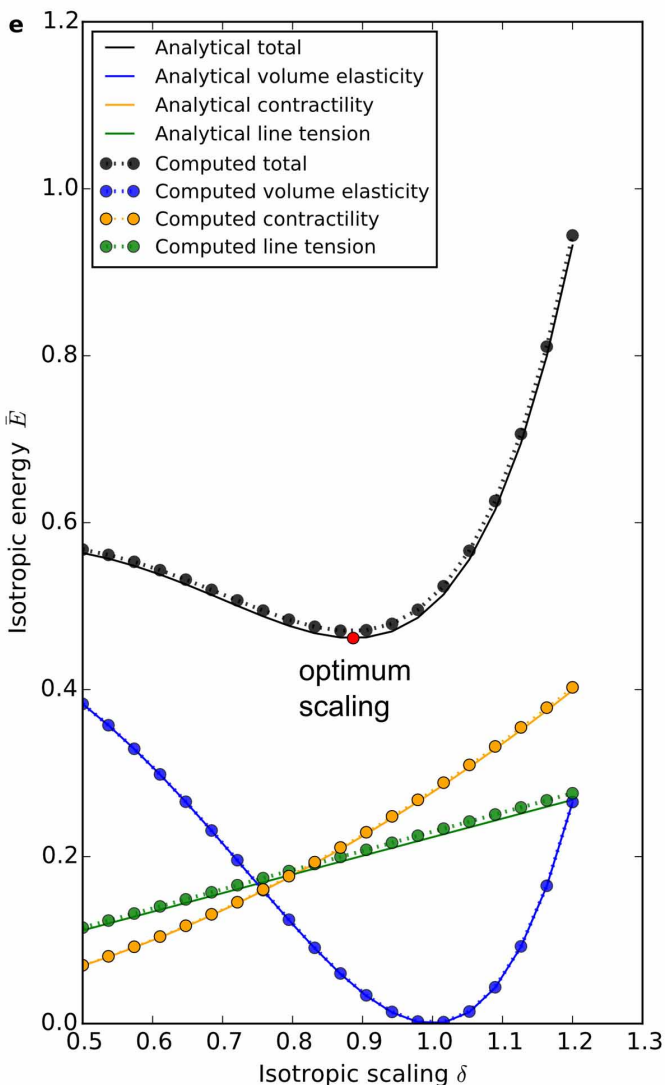

similar trend of growing area with the number of sides, in good quantitative agreement with Farhadifar et al..$^{15}$. c, Distribution of the number of neighbouring cells (or, equivalently, of the number of cell sides), to be compared with Fig. $2 \mathrm{f}$ of Farhadifar et al..$^{15}$; once again, we are in good quantitative agreement with their model. d, Ground state diagram of the vertex model, comparing two-dimensional and three-dimensional hexagonal network boundaries (we restricted ourselves to $\Lambda>0$ and $\Gamma>0$ regions). The black dot indicates the chosen values for the line tension and contractility parameters, which are the same as case I in the Farhadifar et al. ${ }^{15}$ article. e, Variation of the normalized energy of a regular epithelium comprised of identical hexagonal cells as a function of a scale factor $\delta$. Plain lines, analytical calculus; dotted line, average cell energy for a cylindrical tissue of 32 cells in diameter per 29 cells long. A scaling of $\delta=1$ means that the cells are at their equilibrium volume in the absence of elasticity and contractility, and thus corresponds to the minimum of the blue lines (volume elasticity). Green lines correspond to line tension and yellow lines to contractility. The discrepancy between theoretical and computed values is due to the effect of cells lying at the border of the cylindrical epithelium. 
a
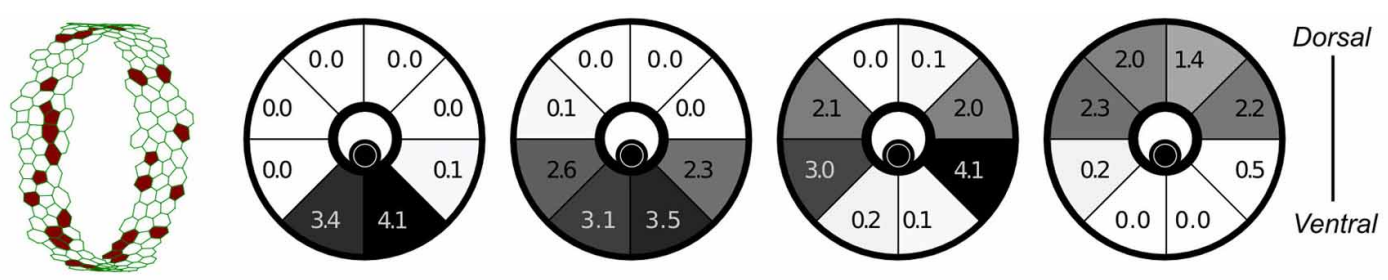

b Number of apoptotic cells 0

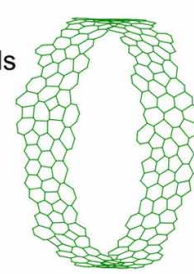

c

5

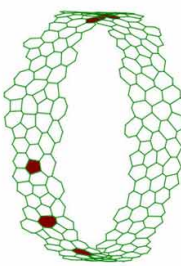

d
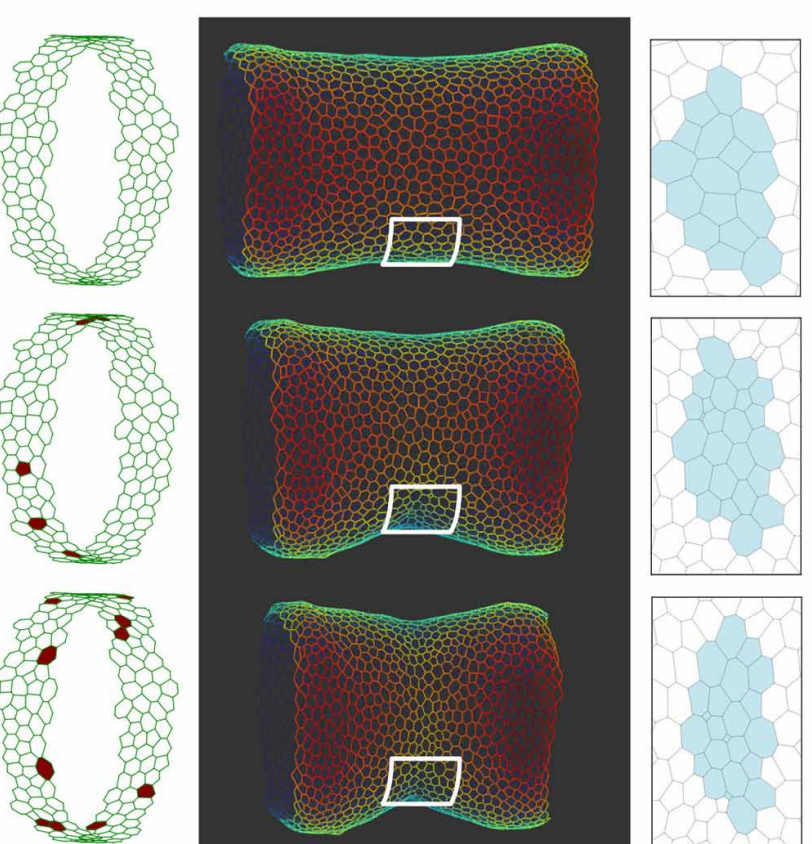

Anisotropy

Normalised area Orientation
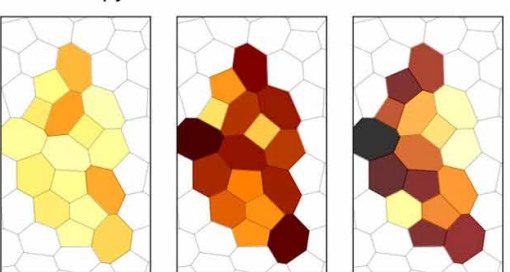

10
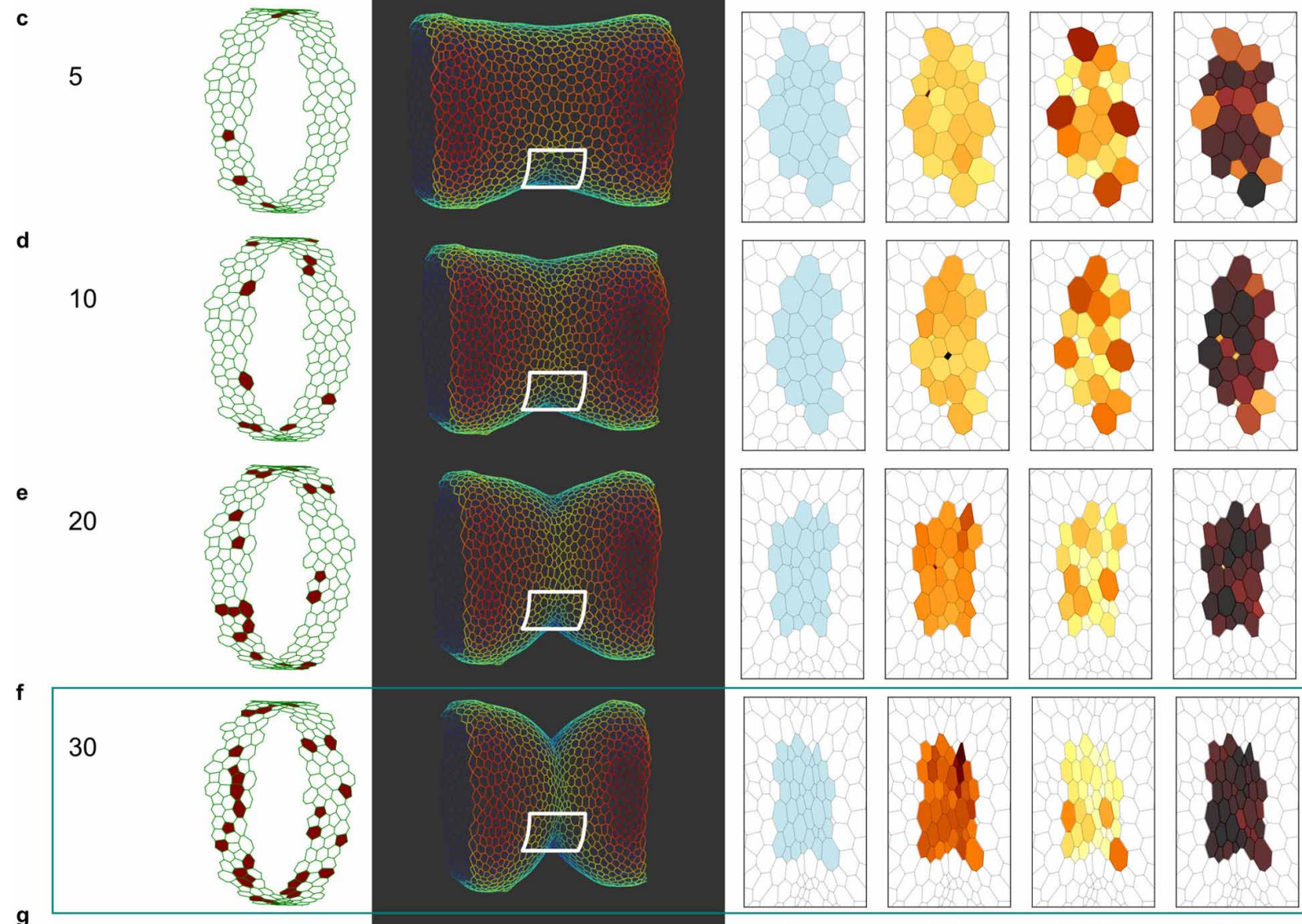

random pattern
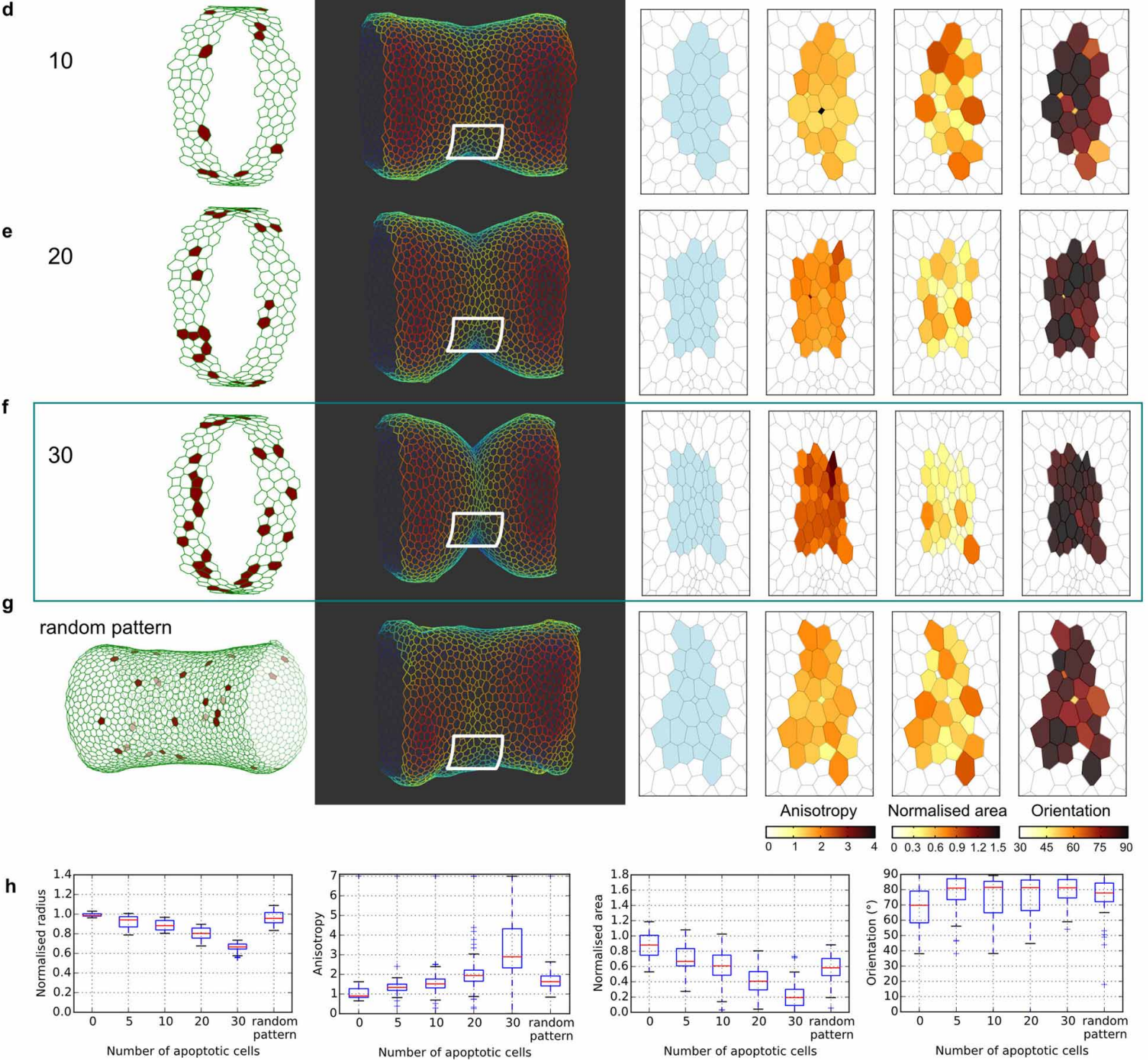
Extended Data Figure 6 Effect of the apoptosis pattern on epithelium folding in silico. This figure is associated with Fig. 3. a, Representation of the in silico cell death pattern. Note the similarity with the in vivo distribution of apoptosis observed in biological samples (Fig. 1b). Nonetheless, the representation is not exactly comparable since cell death pattern is represented relative to a developmental stage in the biological samples, whereas in the model, the cell death pattern is represented relatively to the number of dead cells generated by the theoretical simulation since the time scale is not taken into account in the model. Left, representation of the in silico distribution of apoptotic cells around the fold domain. $\mathbf{b}-\mathbf{g}$, For each panel, from left to right are represented (1) a scheme of the fold domain showing the pattern of apoptosis, (2) a three-dimensional representation showing whole tissue shape and (3) for each condition, the corresponding cell outlines extracted from three-dimensional simulations in which anisotropy, area and orientation of cells from the fold domain are colour-coded. b-f, In silico models showing whole tissue shape with an increasing number of apoptotic cells following the in vivo pattern of apoptosis. Note the gradual increase in anisotropy, gradual decrease of cell area and the preferential orientation with the gradual increase in the number of dying cells. The three-dimensional simulations in $\mathbf{b}$ and $\mathbf{f}$ are those presented in Fig. 3a and Fig. 3c, respectively. The three-dimensional simulation in $\mathbf{f}$ (framed in a blue rectangle in Extended Data Fig. 6, Extended Data Fig. 7 and Extended Data Fig. 8) corresponds to 30 apoptotic cells, with an apico-basal force of $1 \Lambda$ and an increase in apical contractility of $1 \Gamma$. g, In silico model for a random pattern of apoptosis, with all other parameters similar to $\mathbf{f}$. $\mathbf{h}$, The mean value of radius, anisotropy, area and orientation of cells from the whole fold domain, defined as a $\pm 1 \mu \mathrm{m}$ region around the fold centre of simulations $\mathbf{b}-\mathbf{g}$ are represented by box plot. The number of cells considered is $n=33,38,45,61$ and 98 for $0,5,10,20$ and 30 cells, respectively. This number varies due to changes in cells density in the domain. 
a Apico-basal force

$0 \wedge$

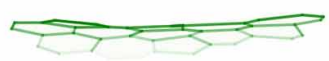

b

$0.5 \wedge$

C
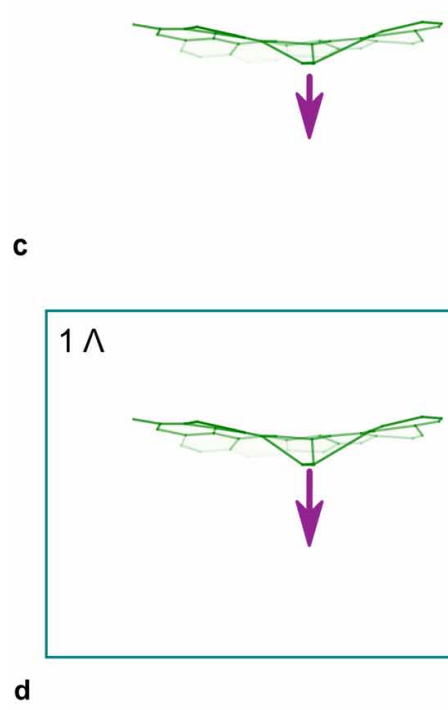

$2 \wedge$

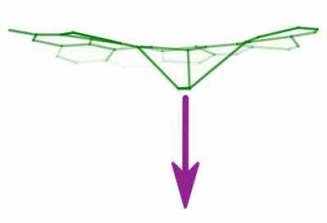

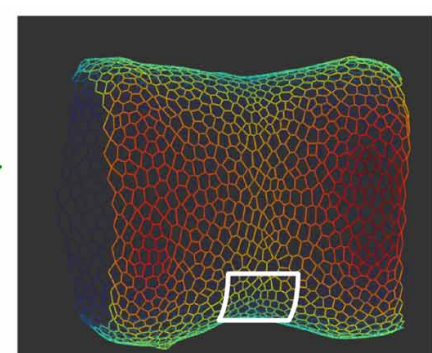

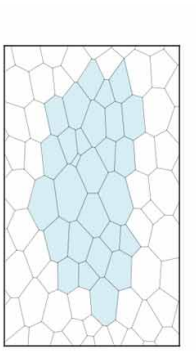

Anisotropy
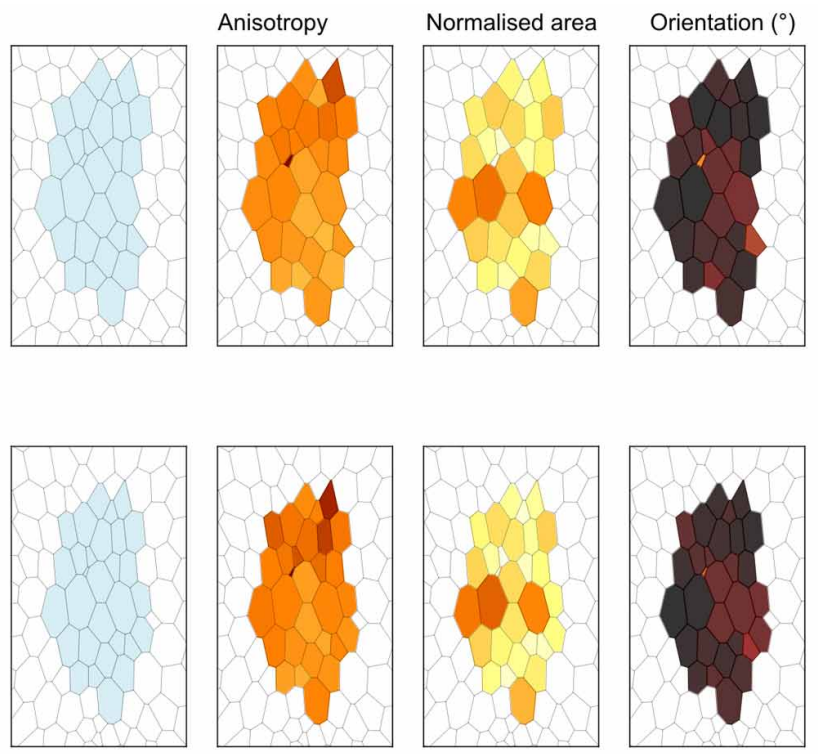
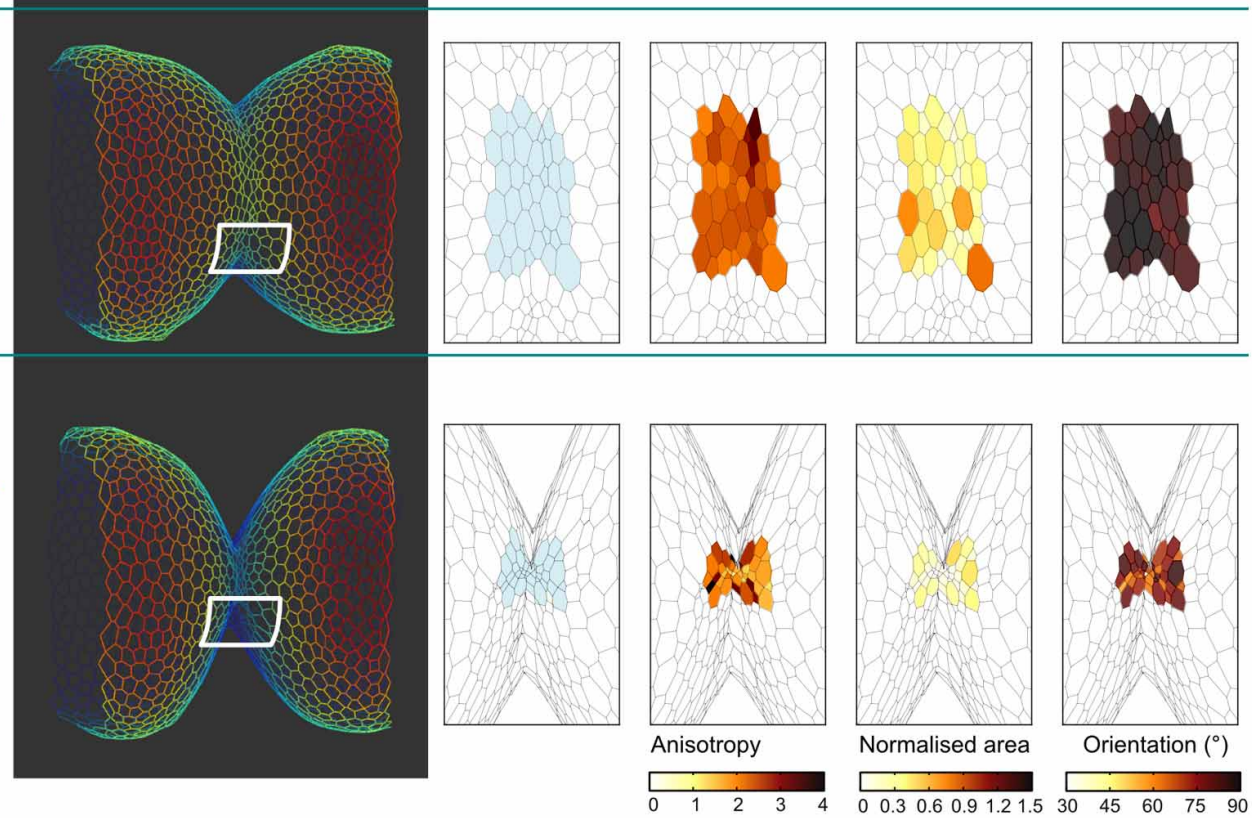

Normalised area

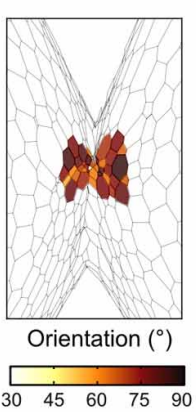

e
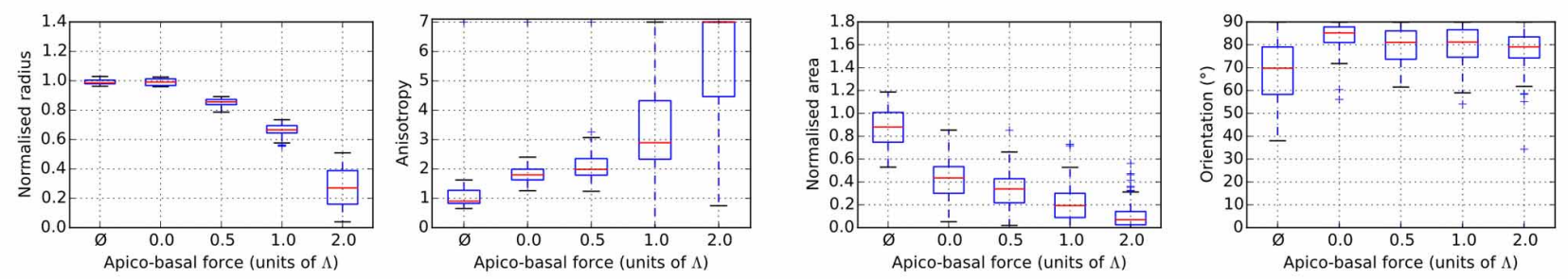

Extended Data Figure $7 \mid$ Effect of apico-basal force intensity on epithelium folding in silico. This figure is associated with Fig. 3. a-d, In silico models showing whole tissue shape following increasing values of apico-basal apoptotic force (30 apoptotic cells, apical contractility: $1 \Gamma$ ). For each panel, from left to right are represented (1) a scheme of the strength of the apico-basal force applied, (2) a three-dimensional representation showing whole tissue shape and (3) for each condition, the corresponding cell outlines extracted from three-dimensional simulations in which anisotropy, area and orientation of

cells from the fold domain are colour-coded. Note the gradual increase in anisotropy, gradual decrease of cell area and the preferential orientation with the gradual increase of the apico-basal force. e, The mean value of radius, anisotropy, area and orientation of cells from the whole fold domain of simulations a-d are represented by box plot. The $\varnothing$ symbol corresponds to the condition in absence of apoptosis (from Extended Data Fig. 6b).

The number of cells considered is $n=60,69,98$ and 157 for $\Lambda=0,0.5$, 1 and 2 , respectively. 
a Contractility increase

$0 \Gamma$

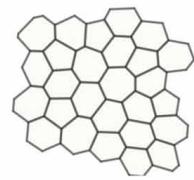

$0.5 \Gamma$

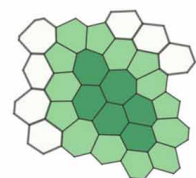

d

$2 \Gamma$

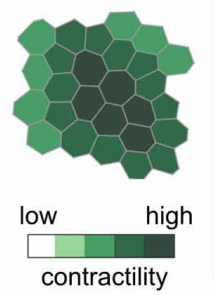

e
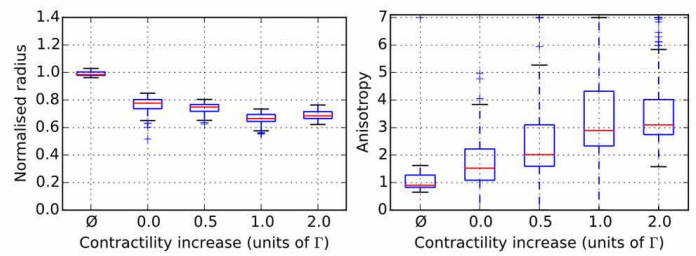

Extended Data Figure $8 \mid$ Effect of the gradual apical cell contractibility increase on epithelium folding in silico. This figure is associated with Fig. 3. a-d, In silico models showing whole tissue shape with an apico-basal force of $1 \Lambda$ in 30 apoptotic cells and increasing values of apical contractility in neighbouring cells. For each panel, from left to right are represented (1) a scheme representing the gradual increase of contractility values applied in apoptotic neighbours, (2) a three-dimensional representation showing whole tissue shape and (3) for each condition, the corresponding cell outlines extracted from three-dimensional simulations in which cell anisotropy, area
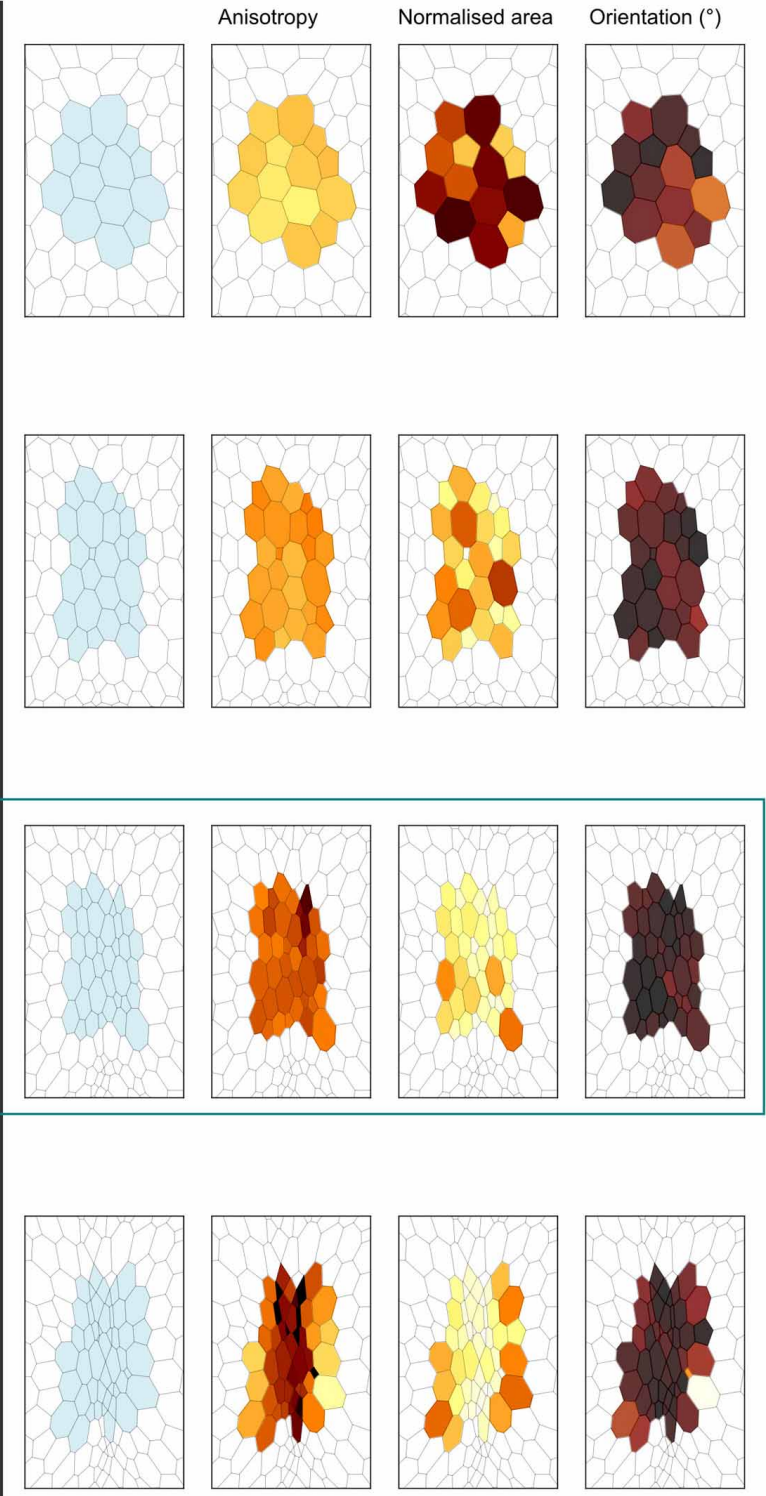

Anisotropy

Normalised area

Orientation
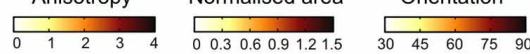
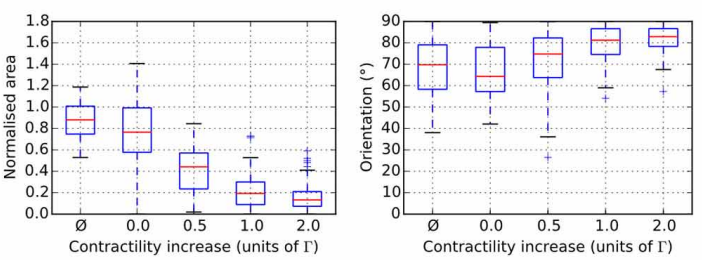

and orientation from the fold domain are colour-coded. Note the gradual increase in anisotropy, gradual decrease of cell area and the preferential orientation with the gradual increase of contractility. e, The mean value of radius, anisotropy, area and orientation of cells from the whole fold domain of simulations a-d are represented by box plot. The $\emptyset$ symbol corresponds to the condition in absence of apoptosis (from Extended Data Fig. 6b). The number of cells considered is $n=31,61,98$ and 137 for $\Gamma=0,0.5$, 1 and 2 , respectively. 
a

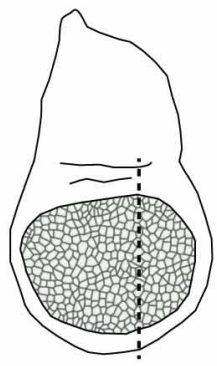

control

(hs::flp, act:FRT-STOP-FRT:Gal4, uas::GFP $\mathrm{X}$ uas::GFP, uas::rpr; no induction)

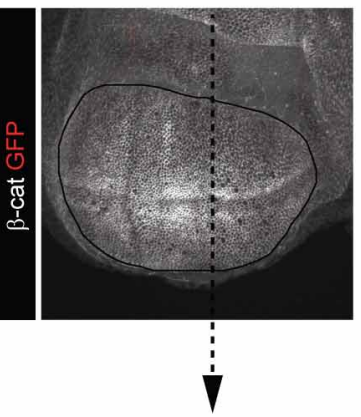

b

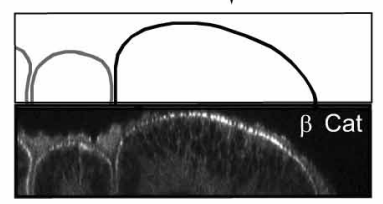

c

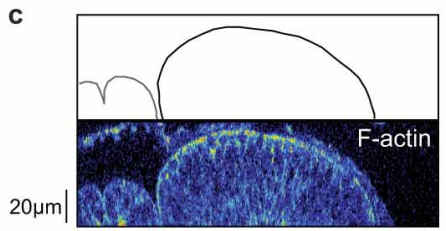

e

control

(ptc::Gal4, uas::GFP)

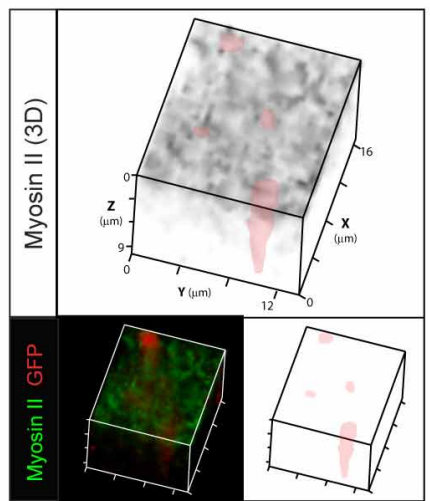

$a^{\prime}$

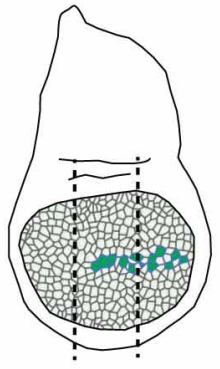

ectopic cell death

(hs.:flp, act:FRT-STOP-FRT:Gal4, uas.:GFP

$X$ uas.:GFP, uas.:rpr; induction)

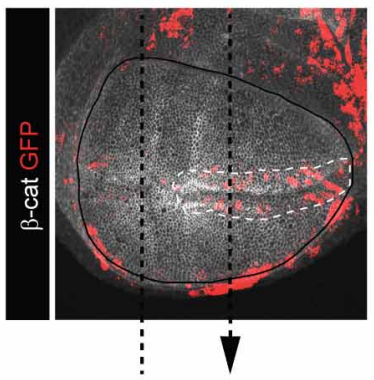

b'

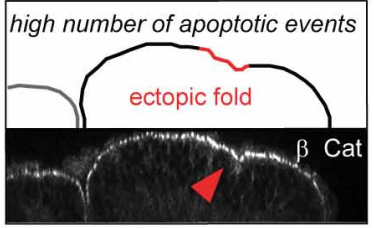

i

b"'

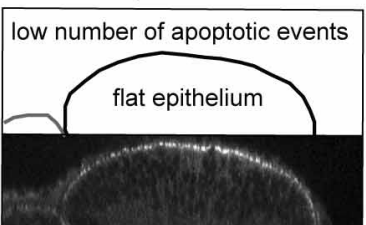

$c^{\prime}$

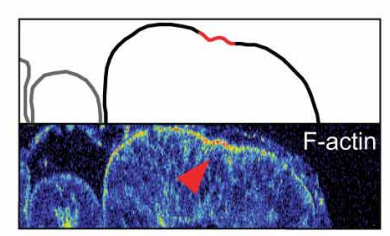

e

ectopic cell death

(ptc::Gal4, uas:::GFP X uas::GFP, uas::rpr)

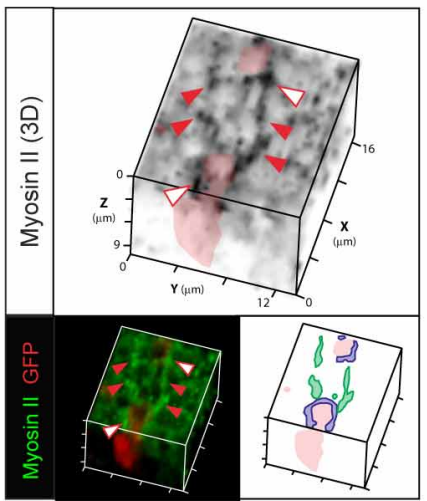

a"

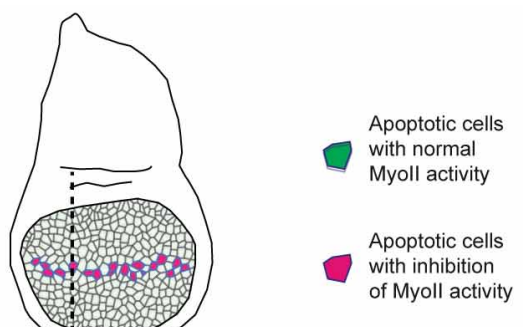

ectopic cell death + DN-Myoll (hs:.flp, act:FRT-STOP-FRT:Gal4, uas.:GFP

$\mathrm{X}$ uas.:DN-zip-GFP, uas.:hid ; induction)
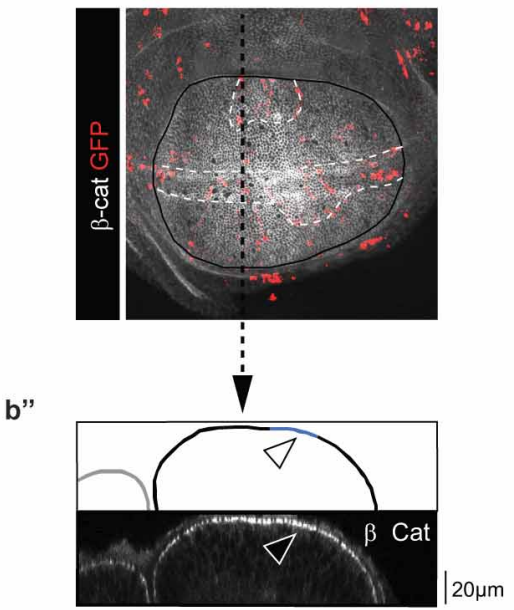

d

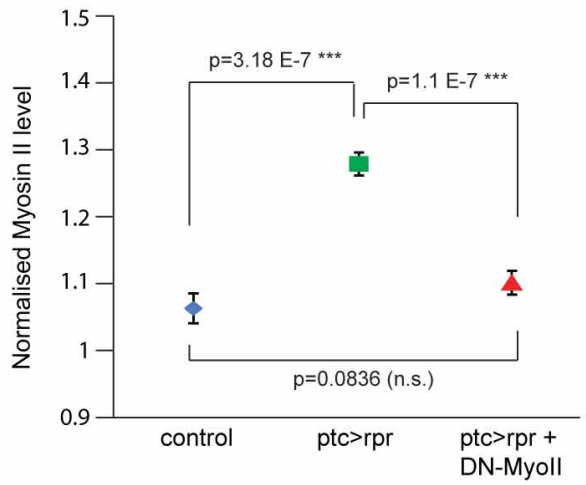

e"

ectopic cell death + DN-Myoll

(ptc::Gal4, uas::GFP X uas:::DN-zip-GFP, uas::rpr)

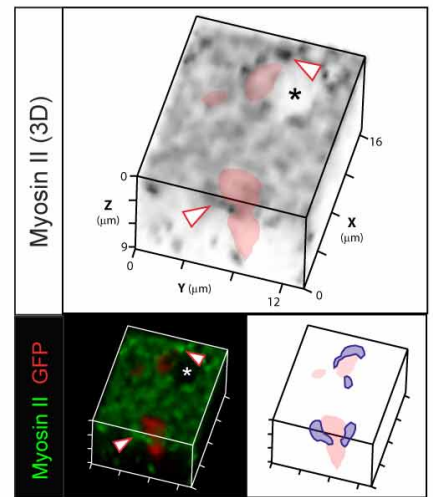

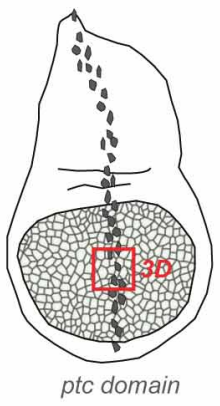


Extended Data Figure 9 | Fold formation as a result of apoptotic forces. This figure is associated with Fig. 4. a-a' ${ }^{\prime \prime}$, Schematics of wing discs depicting the pattern of apoptosis and the presence or absence of myosin II activity in apoptotic cells in each genetic context analysed in $\mathbf{b}-\mathbf{b}^{\prime \prime \prime}$ and $\mathbf{c}-\mathbf{c}^{\prime}$. Control (a, b, y,w,hs::flp; act-frt-y+-frt::Gal4, uas::GFP / uas::lifeactGFP; uas::rpr without clones), Ectopic cell death ( $\mathbf{a}^{\prime}, \mathbf{b}^{\prime}, \mathbf{b}^{\prime \prime \prime}$, y,w,hs::flp; act-frt-y+-frt::Gal4, uas::GFP / uas:::lifeactGFP; uas::rpr), and ectopic cell death without myosin II activity specifically in apoptotic cells $\left(\mathbf{a}^{\prime \prime}, \mathbf{b}^{\prime \prime}, \mathrm{y}, \mathrm{w}, \mathrm{hs}::\right.$ flp; uas::DN-zip::GFP, uas::hid; act-frt-CD2-frt::Gal4). Note that, in the latter condition, myosin II activity is maintained in living cells. Wing discs were dissected from larvae heat shocked for $20 \mathrm{~min}$ at $38^{\circ} \mathrm{C}$. b- $-\mathbf{c}^{\prime}$, For each panel, sagittal views and schematics of sagittal sections are shown (sagittal views correspond to the black dotted line indicated in $\mathbf{a}-\mathbf{a}^{\prime \prime}$ ). $\mathbf{b}-\mathbf{b}^{\prime \prime \prime}$, A high concentration of myosin II positive apoptotic cells is sufficient to induce a fold in a naive tissue (red arrowhead, $\mathbf{b}^{\prime}, n=11$ ) as shown by the visualization of the wing disc apical surface stained with an anti- $\beta$-catenin antibody (compare $\mathbf{b}^{\prime}$ with $\mathbf{b}$ ). Note that no ectopic fold is observed when only a low number of apoptotic events occur $\left(\mathbf{b}^{\prime \prime \prime}\right)$ or when myosin II is inhibited in apoptotic cells (open arrowhead, $n=5$ out of 6$)\left(\mathbf{b}^{\prime \prime}\right) . \mathbf{c}-\mathbf{c}^{\prime}$, F-actin accumulates in ectopic folds (red arrowhead) when apoptotic myosin II is active in ectopic dying cells (compare $\mathbf{c}^{\prime}$ with c, $n=3$ ). d, Quantification of myosin II levels in the patch ( $p t c)$ domain (domain of cell death induction) in control (w; ptc::Gal4, uas::GFP; tub::G80[ts] / SM5-TM6B) ; ptc > rpr (w; ptc::Gal4, uas::GFP / uas::lifeactGFP; tub::G80[ts] / uas::rpr) and ptc $>$ rpr+DN-MyoII (w; ptc::Gal4, uas::GFP / uas::DNzip::GFP; tub::G80[ts] / uas::rpr) wing discs (control, $n=24$; ptc $>$ rpr, $n=28$; ptc $>\operatorname{rpr}+$ DN-MyoII, $n=32$ ). Values are represented as mean values with error bars representing standard errors. The intensity of the signal in the $p t c$ domain has been normalized with the intensity in the anterior and posterior domains of the same disc (n.s. is for non-significant). We used the nonparametric Wilcoxon rank sum test (also called Mann and Whitney test). e-e", Wing disc close-ups (of wing discs shown in Fig. 4c) and schematics in the absence (e) or presence ( $\mathbf{e}^{\prime}$ and $\mathbf{e}^{\prime \prime}$ ) of ectopic apoptosis in the ptc domain (red cells, false-coloured in red on the black and white images), with ( $\left.\mathbf{e}^{\prime}\right)$ or without ( $\left.\mathbf{e}^{\prime \prime}\right)$ myosin II activity in dying cells. $\mathbf{e}^{\prime}$, Note that we can distinguish two distinct pools of stabilized apical myosin II: "contractile ring myosin II" required for dying cell extrusion ${ }^{16}$ (blue arrows, purple in schematics) and "fold domain apical myosin II" stabilized in response to the apico-basal apoptotic force (red arrows, green in schematics). $\mathbf{e}^{\prime \prime}$, Note that, consistently with normal extrusion in this background, contractile ring myosin II is still present around apoptotic cells (blue arrows), whereas fold domain apical myosin II is absent. The star points at a dividing cell, further indicating that myosin II is still present in apoptotic cell neighbours. 


\section{RESEARCH LETTER}

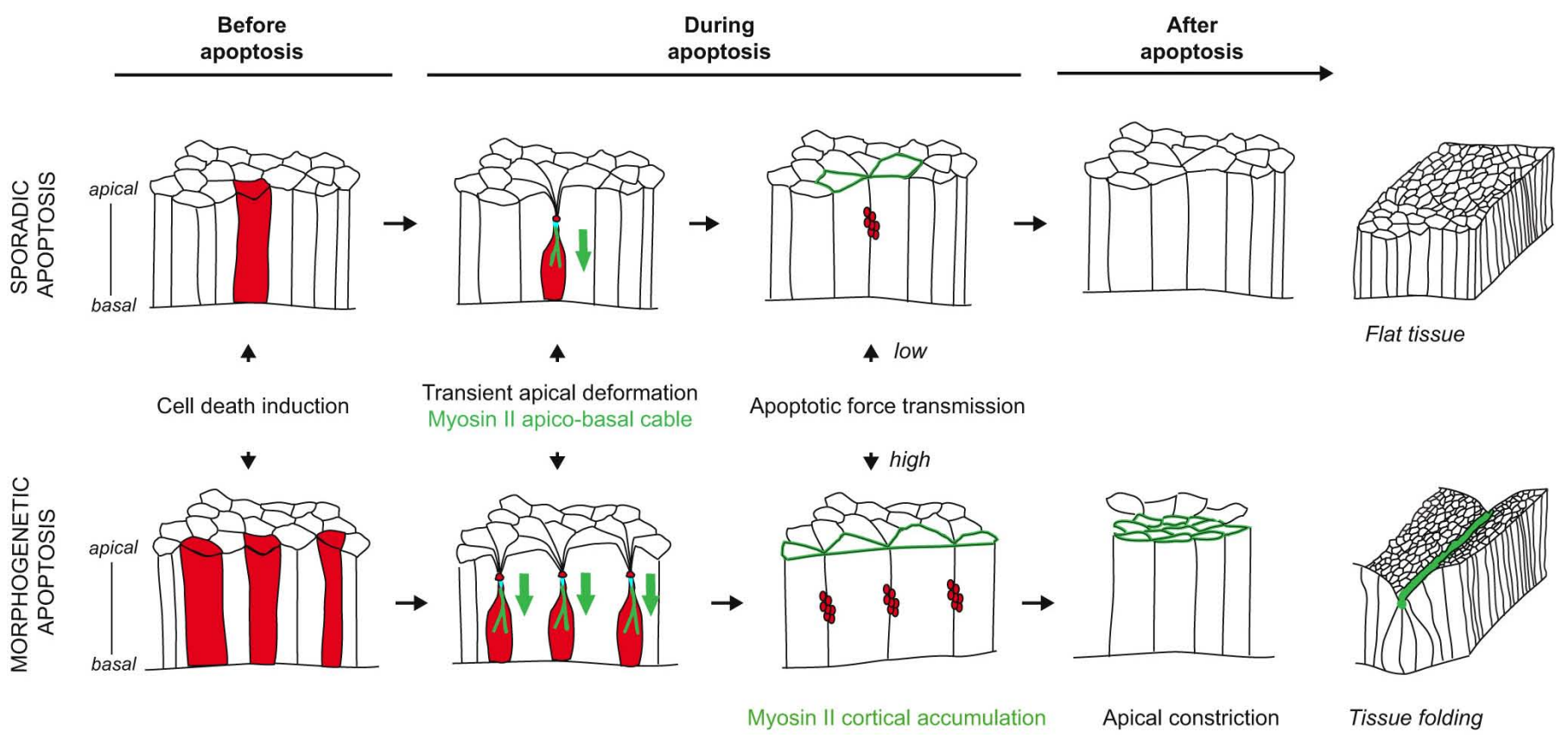

Extended Data Figure $10 \mid$ Model of apoptosis-dependent epithelium folding. 